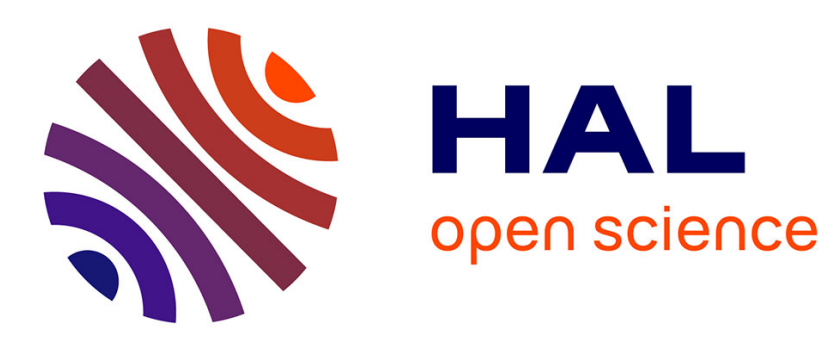

\title{
First-principles calculations of carbon clathrates: Comparison to silicon and germanium clathrates \\ Damien Connetable
}

\section{To cite this version:}

Damien Connetable. First-principles calculations of carbon clathrates: Comparison to silicon and germanium clathrates. Physical Review B: Condensed Matter and Materials Physics (1998-2015), 2010, 82 (7), 10.1103/PhysRevB.82.075209 . hal-02325525

\section{HAL Id: hal-02325525 \\ https://hal.science/hal-02325525}

Submitted on 22 Oct 2019

HAL is a multi-disciplinary open access archive for the deposit and dissemination of scientific research documents, whether they are published or not. The documents may come from teaching and research institutions in France or abroad, or from public or private research centers.
L'archive ouverte pluridisciplinaire HAL, est destinée au dépôt et à la diffusion de documents scientifiques de niveau recherche, publiés ou non, émanant des établissements d'enseignement et de recherche français ou étrangers, des laboratoires publics ou privés. 


\title{
First-principles calculations of carbon clathrates: Comparison to silicon and germanium clathrates
}

\author{
Damien Connétable* \\ CIRIMAT UMR 5085, CNRS/INP/UPS, Ecole Nationale d'Ingénieurs en Arts Chimiques et Technologiques (ENSIACET) 4, \\ allée Émile Monso, BP 44362, F-31030 Toulouse Cedex 4, France
}

(Received 19 December 2009; revised manuscript received 18 June 2010; published 26 August 2010)

\begin{abstract}
We employ state-of-the-art first-principles calculations based on density-functional theory and densityfunctional perturbation theory to investigate relevant physical properties and phase diagram of the free guest type-I $(X-46)$ and type-II $(X-34)$ carbon clathrates. Their properties and those of silicon and germanium diamonds, and clathrates have been computed and compared within the same approach. We briefly present and discuss their structural, cohesive, and electronic properties. In particular, we present different results about electronic properties of carbon clathrates. From the symmetry analysis of electronic states around the band gap, we deduce their optical properties, and we forecast the effects of hypothetical-doped elements on their electronic band gap. We then report first-principles calculations of vibrational, thermodynamical, and elastic properties. Whereas vibrational properties of $\mathrm{Si}$ and $\mathrm{Ge}$ systems can be linked through their atomic weight ratio, we show that the vibrational properties of carbon structures differ strongly. Raman and infrared spectra of all clathrates are also calculated and compared. The effects of pressure and temperature on thermodynamical properties (heat capacity, entropy, thermal expansion, etc.) within static and quasiharmonic approximations are investigated. It is shown that thermodynamical properties of carbon clathrates and diamond present a similar evolution up to high pressures $(100 \mathrm{GPa})$ and over a large range of temperatures $([0,1500] \mathrm{K})$. Then we deduce the equilibrium phase diagram $(P, T)$ of $\mathrm{C}-2 / \mathrm{C}-34 / \mathrm{C}-46$. We conclude the paper with a presentation of elastic properties computed from acoustic slopes.
\end{abstract}

DOI: 10.1103/PhysRevB.82.075209

\section{INTRODUCTION}

Clathrates have emerged these last decades and have appeared to belong to be a family of compounds with high potentials. The main feature of clathrates is the possibility of tuning their structures: either strongly doped systems (until $20 \%$ ) or substituted systems. Their properties are then correlated with this tuning: a high thermoelectrical power, ${ }^{1-4}$ adapted electronic features (either $n, p$, or both type semiconductors in function to the intercalated and substituted elements),,$^{5-8}$ a large electron-phonon coupling,,${ }^{9,10}$ high stability under pressure. ${ }^{11}$ Some clathrates present several of these properties: one can cite, for example, the barium or the hypothetical iodine-doped silicon clathrates. ${ }^{5}$ Doping elements are changing the carrier concentration in free guest clathrates intrinsic bands and modifying the position of the Fermi level in the system. Similarly, carbon element exists under a large variety of allotropic structures: from threedimensional (diamond, Si-C, B-C-N alloys, etc.), twodimensional (graphene, graphite, intercalated graphene), one-dimensional (nanotubes), to zero-dimensional networks (fullerenes, clusters). Each of these structures exhibits actual or potential applications in diverse applied fields.

Carbon clathrates should combine the originality of clathrates (tuning, high doping) with a light element. Due to their speculative existence, the literature on carbon clathrates is not exhaustive. The published studies deal with their lattice parameters, ${ }^{12-15}$ bulk modulus, stability, phase diagram, ${ }^{13,16}$ electronic band structures, ${ }^{17,18}$ plastic features, ${ }^{19}$ and about their potential superconductivity. ${ }^{10,18,20,21}$ Studies of their fundamental vibrational and associated properties remain nevertheless limited within a full density-functional-theory (DFT) approach. As it has been shown, carbon clathrates
PACS number(s): 82.75.-z, 78.30.Na, 63.20.-e, 65.40.De should yield large critical temperature and have a hardness similar to diamond. It is also significant to explore any special features in the thermal expansion, heat capacity, Raman, infrared spectra, and phase diagram of these unusual expanded-framework semiconducting crystals.

The purpose of this paper is to present and discuss, by means of a first-principles approach, the properties of two empty carbon clathrates -C-34 and C-46-, and to compare them to other empty clathrates of group IV ( $\mathrm{Si}$ and $\mathrm{Ge}$ ). The first part of the manuscript is briefly devoted to the structural, energetic, and electronic properties, which are presented and compared to those of the literature. An analysis of symmetries of electronic states around the band gap of carbon clathrates is in particular displayed. In the second part, using the density-functional perturbative theory, we present the properties calculated from dynamical properties: an analysis of phonon spectra, of Raman and infrared intensities, a study of the effect of temperature and pressure on thermodynamical properties and the $(T, P)$ phase diagram of C-2/C-34/C-46. We conclude by a discussion on their elastic properties.

\section{PRESENTATION}

Clathrates are composed of polyhedral cages which are sharing their faces. The atoms are linked together through $s p^{3}$-type covalent bonds. The nearest-neighbor distances and the angles between bonds are approximately the same as those found in the diamond systems. Their main difference with diamonds is the presence of $87 \%$ of pentagonal cycles, where there are only hexagonal cycles. These pentagonal cycles are expected to be at the origin of the specific electronic and dynamical properties of clathrates. ${ }^{22}$ Besides, the originality of cagelike materials is the possibility of a huge 
TABLE I. Wyckoff positions of type-I and type-II clathrates.

\begin{tabular}{lccc}
\hline \hline \multicolumn{2}{c}{$X-34(F d \overline{3} m:$ No 227) } & \multicolumn{2}{c}{$X-46(P m \overline{3} n:$ No 223) } \\
\hline $8 a(2)$ & $x, y, z=0$ and $=1 / 4$ & $6 c$ & $x=1 / 4 ; y=0 ; z=1 / 2$ \\
$32 e(8)$ & $x, y, z=-0.092$ & $16 i$ & $x, y, z=0.184$ \\
$96 g(24)$ & $x, y=-0.058 ; z=0.246$ & $24 k$ & $x=0 ; y=0.308 ; z=0.117$ \\
\hline \hline
\end{tabular}

endohedral doping. Clathrates can thus be doped either by inserting (intercalation) until one atom in each cage, which corresponds to $20 \%$ of doping, or by substitution. Doping of clathrates is thus much easier than doping of the diamond phase (only some percent by substitution).

The type-I clathrate, namely, $X-46$, has a simple cubic lattice with 46 atoms per unit cell and the $P m \overline{3} n$ (No. 223) group symmetry (see Table I). The type-II clathrate, called $X-34$ or $X-136$, is a face center cubic lattice with 34 atoms in the unit cell and the $F d \overline{3} m$ (No. 227) symmetry.

\section{THEORETICAL FRAMEWORK}

Calculations have been performed using the DFT-based QUANTUM-ESPRESSO package (Ref. 23). The local density approximation, through the Perdew-Zunger parameterization ${ }^{24}$ of the Ceperley-Alder exchange correlation functional, ${ }^{25}$ and norm-conserving Troullier-Martins ${ }^{26}$ pseudopotentials factorized in Kleinman-Bylander form ${ }^{27}$ have been employed. To optimize the structures, the Brillouin zone has been sampled by a $2 \times 2 \times 2$ Monkhorst-Pack grid $^{28}$ which corresponds to one and two irreducible $k$ points for $X-46$ and $X-34$, respectively. The wave functions are expanded on plane-wave basis with kinetic energies up to a real-space grid with 50 Ryd (for carbon) and 20 Ryd (for germanium and silicon) energies cutoff. For the electronic density of states $(e \mathrm{DOS})$ and the formation energies, we have adopted finest grids $(10 \times 10$ $\times 10$ ) with higher energies cutoff (60 Ryd for carbon and 30 Ryd for Si/Ge systems).

The phonon frequencies have been determined by the dynamical matrix, Born effective charges, and dielectric constants, which have been computed using the linear response theory of the DFT. ${ }^{29,30}$ In order to generate the force constants matrices and carry out the inverse Fourier transformation, $2 \times 2 \times 2 \mathbf{q}$ grids have been employed for static properties (with $6 \times 6 \times 6 \mathbf{k}$ grids mesh, and 50 and 20 Ryd of energies cutoff for carbon and silicon/germanium systems, respectively). For diamond phases, we have increased the $\mathbf{k}$ and $\mathbf{q}$ grids $(15 \times 15 \times 15$ and $8 \times 8 \times 8$, respectively). The vibrational density of states has been calculated on $20 \times 20$ $\times 20$ grids with the tetrahedron method.

\section{STRUCTURAL AND ELECTRONIC PROPERTIES}

In the Table II we report the optimized lattice parameters, the bulk modulus (fitted by a Murnaghan's equation of state $\left.^{37}\right)$ as well as the cohesive energies of the corresponding species $(X=\mathrm{C}, \mathrm{Si}$, and $\mathrm{Ge})$ and crystallographic structures $(X-2=$ diamond, $X-34$, and $X-46)$. These results are found in good agreement with the experimental data and theoretical
TABLE II. Carbon, silicon, and germanium diamond and clathrates phases lattice parameters (in $\AA$ ), bulk modulus (in GPa), and formation energy (in meV/atom). The evolution of the atomic volume taken as reference diamond phase $\delta V$ is given $\left[\delta V=V_{\text {at }}(X-2) / V_{\text {at }}(\right.$ system $\left.)\right]$.

\begin{tabular}{lcccc}
\hline \hline System & $a_{o}$ & $\delta V$ & $B_{o}$ & $E_{f}$ \\
\hline $\mathrm{C}-2$ & $3.58\left(3.57^{\mathrm{a}}\right)$ & 1.00 & $460\left(545^{\mathrm{a}}\right)$ & 0 \\
$\mathrm{C}-34$ & $9.60\left(9.56^{\mathrm{b}}\right)$ & 0.86 & $393\left(398^{\mathrm{b}}\right)$ & $150\left(134^{\mathrm{c}}\right)$ \\
$\mathrm{C}-46$ & $6.65\left(6.80^{\mathrm{d}}\right)$ & 0.87 & $395\left(398^{\mathrm{e}}\right)$ & $110\left(144^{\mathrm{c}}\right)$ \\
$\mathrm{Si}-2$ & $5.41\left(5.43^{\mathrm{a}}\right)$ & 1.00 & $97\left(99^{\mathrm{a}}\right)$ & 0 \\
$\mathrm{Si}-34$ & $14.53\left(14.62^{\mathrm{f}}\right)$ & 0.87 & $86\left(84^{\mathrm{g}}\right)$ & $60\left(80^{\mathrm{g}}\right)$ \\
$\mathrm{Si}-46$ & $10.08\left(10.15^{\mathrm{g}}\right)$ & 0.88 & $87\left(84^{\mathrm{g}}\right)$ & $70\left(90^{\mathrm{g}}\right)$ \\
$\mathrm{Ge}-2$ & $5.58\left(5.66^{\mathrm{a}}\right)$ & 1.00 & $75\left(77^{\mathrm{a}}\right)$ & 0 \\
$\mathrm{Ge}-34$ & $15.02\left(15.13^{\mathrm{h}}, 15.21^{\mathrm{i}}\right)$ & 0.88 & $68\left(67^{\mathrm{j}}, 61^{\mathrm{h}}\right)$ & $40\left(50^{\mathrm{h}}\right)$ \\
$\mathrm{Ge}-46$ & $10.40\left(10.47^{\mathrm{h}}\right)$ & 0.89 & $68\left(68^{\mathrm{j}}, 62^{\mathrm{h}}\right)$ & $50\left(50^{\mathrm{h}}\right)$ \\
\hline \hline
\end{tabular}

${ }^{\mathrm{a}}$ Reference 31 .

${ }^{\mathrm{b}}$ Reference 17.

${ }^{\mathrm{c}}$ Reference 12 .

${ }^{\mathrm{d}}$ Reference 20.

eReference 19.

${ }^{\mathrm{f}}$ Reference 32.
g Reference 33.
${ }^{\mathrm{h}}$ Reference 34.
${ }^{\mathrm{i}}$ Reference 35.
${ }^{\mathrm{j}}$ Reference 36.

predictions for the germanium (see Refs. 34 and 36), silicon (Refs. 12, 22, 32, and 33), and carbon elements (Refs. 12-14, 17, 19, and 20).

The electronic band structures $(e \mathrm{BS})$ and $e \mathrm{DOS}$ of carbon and silicon systems are plotted in Fig. 1 (electronic properties of germanium clathrates are not presented here). We found that all empty clathrates are semiconductors with a large gap. Band gaps $\left(\Delta_{\text {gap }}\right.$, Table III) are found greater in $\mathrm{Ge} / \mathrm{Si}$ clathrates than in diamond phases, contrary to carbon clathrate, in agreement with previous DFT values. ${ }^{17,32,36}$

We provide a qualitative interpretation of the variation in this electronic band gap for silicon and carbon elements (the case of germanium is different due to the presence of $d$ core states): from chemical and crystallographic arguments. Gaps in $\Gamma$ are found smaller in clathrates than in diamonds. Since

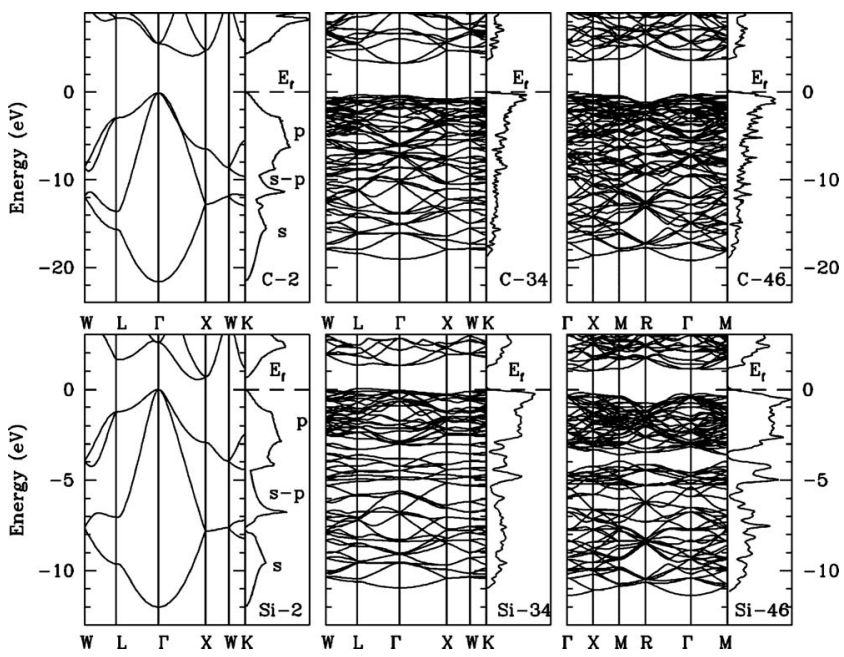

FIG. 1. Electronic band structures (in eV) and density of states. The Fermi level has been put at the top of the valance band. 
TABLE III. Band gaps $\left(\Delta_{\text {gap }}\right)$ in eV.

\begin{tabular}{lcc}
\hline \hline $\mathrm{C}-2$ & $\mathrm{C}-34$ & $\mathrm{C}-46$ \\
$3.9\left(5.48^{\mathrm{a}}\right)$ & $3.7\left(5.15^{\mathrm{b}}\right)$ & $3.7\left(5.25^{\mathrm{b}}\right)$ \\
$\mathrm{Si}-2$ & $\mathrm{Si}-34$ & $\mathrm{Si}-46$ \\
$0.62\left(1.17^{\mathrm{a}}\right)$ & $1.25\left(1.85^{\mathrm{b}}, 1.9^{\mathrm{d}}\right)$ & $1.3\left(1.9^{\mathrm{b}}\right)$ \\
$\mathrm{Ge}-2$ & $\mathrm{Ge}-34$ & $\mathrm{Ge}-46$ \\
$0.34\left(0.74^{\mathrm{a}}\right)$ & $0.95\left(0.80^{\mathrm{c}}\right)$ & $1.4\left(1.46^{\mathrm{e}}, 1.31^{\mathrm{c}}\right)$ \\
\hline \hline
\end{tabular}

${ }^{\mathrm{a} E x p e r i m e n t ~(R e f . ~ 31) . ~}$

${ }^{\mathrm{b}} \mathrm{GW}$ simulations (Refs. 17 and 18).

${ }^{\mathrm{c} D F T}$ simulation (Ref. 36).

${ }^{\mathrm{d}}$ Experiment (Ref. 38).

eDFT simulation (Ref. 39).

clathrates are highly isotropic systems comparatively to the diamond, the dispersion of the electronic bands is weak outside $\Gamma$ (flat bands). Moreover, in the case of carbon, shells are less sensitive than silicon to the crystallographic environment (no internal $p$ shells, and the system is more compact), the band gap is smaller in carbon clathrates than in diamond. Contrarily, silicon is more affected by its crystallographic environment and the band gap is then reduced in diamond.

For sake of completeness, the irreducible representation of electronic states around band gaps have been analyzed, as already done for silicon clathrates. ${ }^{8}$ For a given structure, the symmetries are found to be roughly the same for carbon and silicon clathrates: along different crystallographic directions, the states of the bottom of conduction bands and the top of valence bands have the same symmetries (see Table IV at $\Gamma$ ). Only some states, near the top of the valence bands, change. From these results and from our previous analysis of silicon clathrates, we deduce that, for carbon clathrates, the optical transitions should be forbidden by means of symmetries in a dipole approximation. Furthermore, the effect of doped elements on electronic properties of carbon clathrates can be forecast by symmetry. Only the bottom of the conduction

TABLE IV. Symmetry classification of the valence and conduction bands around the electronic band gap for $X-46$ and $X-34$ systems where $X=\mathrm{Si}$ and $\mathrm{C}$. The energies (in $\mathrm{eV}$ ) are calculated at the $\Gamma$ point (symmetry $O_{h}$ ). The reference energies have been set at the top of the valence bands in the $\boldsymbol{\Gamma}$ point.

\begin{tabular}{lcc}
\hline \hline & $\mathrm{C}-46$ & $\mathrm{Si}-46$ \\
\hline$E_{v 3}$ & $-0.19\left(E_{u}\right)$ & $-0.11\left(E_{u}\right)$ \\
$E_{v 2}$ & $-0.05\left(T_{1 g}\right)$ & $-0.10\left(T_{2 g}\right)$ \\
$E_{v 1}$ & $0.00\left(A_{1 u}\right)$ & $0.00\left(A_{1 u}\right)$ \\
$E_{c 1}$ & $+4.06\left(T_{1 u}\right)$ & $+1.45\left(T_{1 u}\right)$ \\
$E_{c 2}$ & $+5.34\left(A_{2 g}\right)$ & $+1.82\left(A_{2 g}\right)$ \\
& $\mathrm{C}-34$ & $\mathrm{Si}-34$ \\
\hline$E_{v 3}$ & $-0.43\left(T_{2 u}\right)$ & $-0.10\left(T_{2 u}\right)$ \\
$E_{v 2}$ & $-0.17\left(T_{1 g}\right)$ & $-0.01\left(E_{u}\right)$ \\
$E_{v 1}$ & $0.00\left(E_{u}\right)$ & $0.00\left(T_{1 g}\right)$ \\
$E_{c 1}$ & $+3.63\left(T_{1 u}\right)$ & $+1.46\left(T_{1 u}\right)$ \\
$E_{c 2}$ & $+6.40\left(A_{2 u}\right)$ & $+2.22\left(A_{2 u}\right)$ \\
\hline \hline
\end{tabular}

states should be hybridized by states of the intercalated element. So, the electronic gap should be increased like in silicon clathrates.

\section{DYNAMICAL PROPERTIES}

\section{A. Vibrational properties}

\section{Phonon dispersion curves}

Our results for the phonon-dispersion curves ( $v$ band) of diamond and clathrates (C, Si, and Ge elements) along several symmetry lines together with the corresponding vibrational density of states ( $v$ DOS) are displayed Fig. 2. Numerical values at the $\boldsymbol{\Gamma}$ point are listed in Tables $\mathrm{V}$ and VI for $X-34$ and $X-46$ clathrates, respectively, and compared with literature data. The vibrational spectra of diamond phasesnot reported here-are in agreement with experimental data (discrepancy in frequencies less 1\%). According to the $\mathrm{O}_{h}$ group symmetry and the Wyckoff positions of atoms in the network (see Table I), the symmetry of the vibrations (noted $v \mathrm{SM})$ are the following:

$$
\left\{\begin{array}{c}
\boldsymbol{v} \mathbf{S M}_{6 c}=A_{2 g} \oplus E_{g} \oplus T_{1 g} \oplus T_{2 g} \oplus T_{1 u} \oplus T_{2 u} \\
\boldsymbol{v} \mathbf{S M}_{16 i}=A_{2 g} \oplus A_{1 g} \oplus 2 E_{g} \oplus 3 T_{1 g} \oplus 3 T_{2 g} \oplus \\
A_{1 u} \oplus A_{2 u} \oplus 2 E_{u} \oplus 3 T_{1 u} \oplus 3 T_{2 u} \\
\mathbf{S M}_{24 k}=2 A_{2 g} \oplus 2 A_{1 g} \oplus 4 E_{g} \oplus 4 T_{1 g} \oplus 4 T_{2 g} \\
\oplus A_{1 u} \oplus A_{2 u} \oplus 2 E_{u} \oplus 5 T_{1 u} \oplus 5 T_{2 u}
\end{array}\right.
$$

for $X-46$ and for $X-34$

$$
\left\{\begin{array}{c}
\boldsymbol{v} \mathbf{S M}_{8 a}=T_{1 u} \oplus T_{2 g} \\
\boldsymbol{v} \mathbf{S M}_{32 e}=A_{1 g} \oplus A_{2 u} \oplus E_{g} \oplus E_{u} \oplus T_{1 g} \oplus 2 T_{1 u} \\
\oplus 2 T_{2 g} \oplus T_{2 u} \\
\mathbf{v S M}_{96 g}=2 A_{1 g} \oplus A_{1 u} \oplus A_{2 g} \oplus 2 A_{2 u} \oplus 3 E_{g} \oplus \\
3 E_{u} \oplus 4 T_{1 g} \oplus 5 T_{1 u} \oplus 5 T_{2 g} \oplus 4 T_{2 u}
\end{array}\right.
$$

From selections rules ${ }^{43}$ and the knowledge of symmetries, we have forecast the Raman and infrared frequencies (noted in the tables $R$ and $I R$, respectively). The group theory assumes that, for cubic systems belonging to the $O_{h}$ group symmetry, the $A_{1 g}, E_{g}$, and $T_{2 g}$ irreducible representations could be Raman active while the infrared modes should have the $T_{1 u}$ symmetry.

For carbon clathrates, no theoretical values have been reported, except for the frequencies of active Raman modes in the hypothetical lithium-doped clathrate. ${ }^{16}$ In the case of other elements, theoretical frequencies have been already calculated (see values reported in Tables V and VI). Raman measurements have been only done in the case of Si-34. ${ }^{40,41,44}$ Our results are in excellent agreement with those of the literature.

We note that the vibrational dispersion curves for $\mathrm{Si}$ and Ge clathrate are very close, while $v$ band and $v$ DOS of carbon clathrates differ significantly from these systems. The difference between $\mathrm{Si}$ and $\mathrm{Ge}$ systems can be rationalized using their respective atomic weights, ${ }^{45}$ as already mentioned for diamond. ${ }^{46}$ The interatomic forces of C-34 and C-46 clathrates are different from the other clathrates in spite of a similar $s p^{3}$ hybridization. 

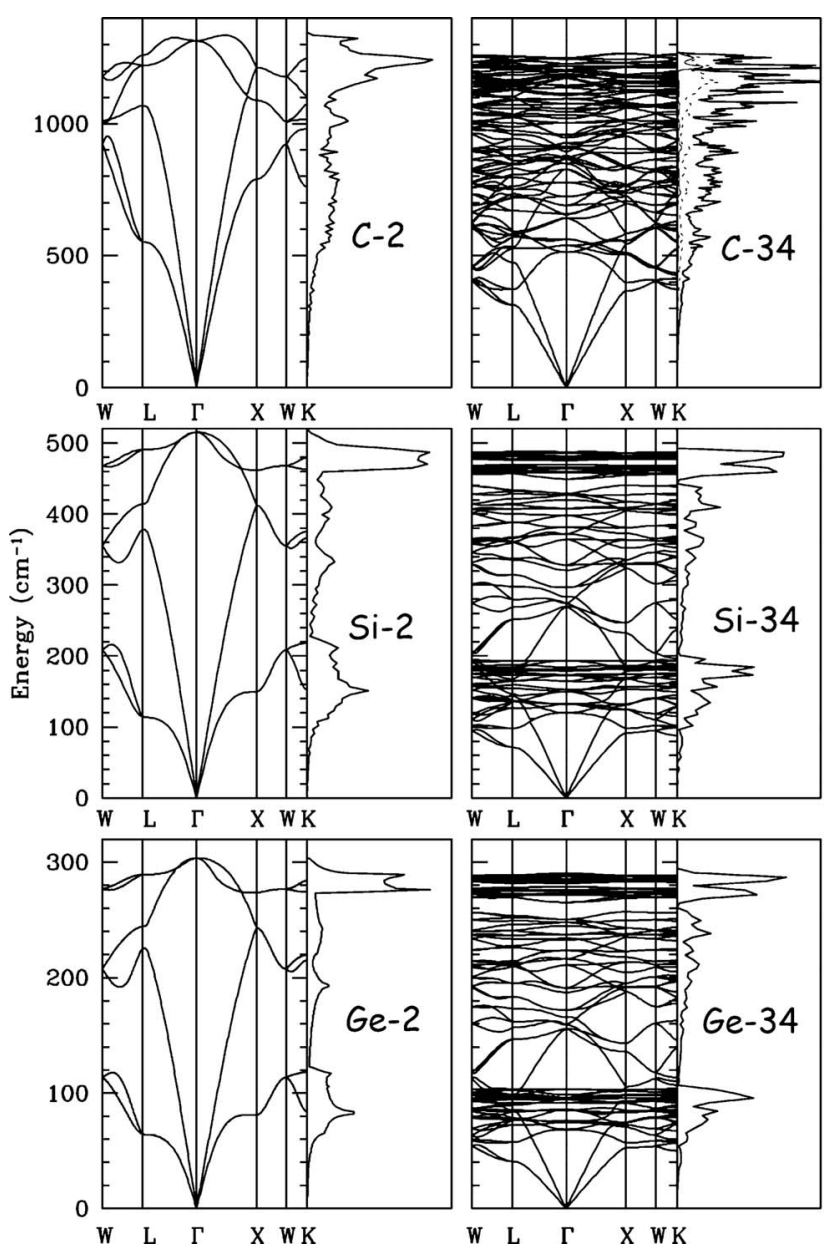

In carbon clathrates, the transverse acoustic modes are no longer flat unlike in other $\mathrm{Si} / \mathrm{Ge}$ systems, which explain why the sharp features of the $v$ DOS (subband) are lacking. As in $\mathrm{Si} / \mathrm{Ge}$ clathrates, most of tetrahedral semiconductors display a peculiar flatness in the transverse acoustic frequencies region (these bands are correlated with long range of inter atomic forces). ${ }^{46}$ In diamond, this difference is less tagged due to its higher compacity.

A comparative analysis of the frequencies in diamond and clathrates reveals that there is in C-34/C-46 two "redshifts" (for optical and acoustic modes) and one "blueshift" in acoustic modes and one "redshift" in optical modes in $\mathrm{Si} / \mathrm{Ge}$ clathrates. The redshift is associated with the weakening of the $X$ - $X$ bonds between clathrates and diamonds. The frustration of the optical modes, due to the presence of pentagonal cycles, induces this shift of $40 \mathrm{~cm}^{-1}$ toward low frequencies in C-34/C-46 and $\mathrm{Si} / \mathrm{Ge}$ clathrates. The number of optical modes, associated with the asymmetric vibrations of two neighbors atoms, decreases with the frustration. We can also note a gap in $\mathrm{Si} / \mathrm{Ge}$ optical frequencies. This gap does not exist in carbon clathrates. The maximum of the optical branches is not located at the zone center for both C-34 and $\mathrm{C}-46$ as in carbon diamond, contrary to other systems, which results in sharp peaks in $v$ DOS for C-34, C-2 and-but less pronounced-for $\mathrm{C}-46$. It is due to the overbending of the optical branches and has been associated, in diamond, ${ }^{47}$ to a sufficiently large interatomic force constants between second-nearest neighbor.
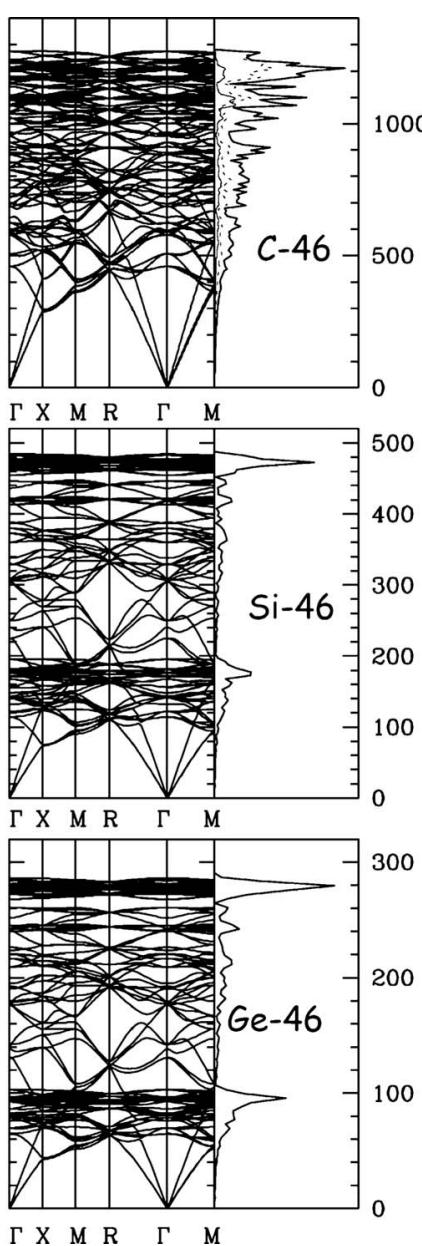

FIG. 2. Vibrational band structures (in $\mathrm{cm}^{-1}$ ) and density of states. We also report the contribution of atoms in $6 c / 16 i$ and $8 a / 32 e$ Wyckoff positions for $\mathrm{C}-46$ and $\mathrm{C}-34$, respectively, in thin lines and dashed lines.

\section{Raman shifts}

Following Ref. 48, the Raman tensor and Raman spectra have been calculated from first-principles approaches. Raman intensities are plotted on Figs. 3 and 4 for type-I and type-II clathrates, respectively. The Raman cross section (at the bottom) is convoluted with a Gaussian broadening and the Raman total intensities of powders are determined following Ref. 49 (Lorentzian convolution). A $10 \mathrm{~cm}^{-1}$ is used as damping constants. The parallel and perpendicular Raman intensities to the incident polarization have been calculated from the depolarization ratio $\rho_{r}\left(\rho_{r}=3 / 4\right.$ for $E_{g}$ and $T_{1 g}$, and $\rho_{r}=0$ for $\left.A_{1 g}\right)$ and added on Figs. 3 and 4 .

As already mentioned, only the Raman spectrum of the free guest Si-34 has been investigated experimentally. ${ }^{40,41,44}$ Raman spectra has been calculated for Si-34 by Dong ${ }^{50}$ and Ge-34/Ge-46 (Ref. 34) using a bond-polarizability model. In Ge systems, the peaks are located at the same positions (shifted, around $10 \mathrm{~cm}^{-1}$ different). In $\mathrm{Si}-34$, three peaks are observed around 120, 250-300, and $470 \mathrm{~cm}^{-1}$, as claimed by Guyot. ${ }^{44}$ The calculated spectra is similar to the experimental spectra obtained by Tang et al. ${ }^{41}$ and Nolas et al. ${ }^{40}$ However, some peaks are missing: in Tang measurements, one $A_{1 g}$ mode at around $320 \mathrm{~cm}^{-1}$, one $T_{2 g}$ mode at $467 \mathrm{~cm}^{-1}$, or in Nolas measurements, the $E_{g}$ and $A_{1 g}$ modes at $454 \mathrm{~cm}^{-1}$ and $480 \mathrm{~cm}^{-1}$, respectively, what makes the comparison sometimes difficult. For example, we arrive to reproduce the vanishing of the $A_{1 g}$ mode in parallel polarized Stokes Ra- 
TABLE V. Frequencies (in $\mathrm{cm}^{-1}$ ) and symmetries of the vibrational modes in $\boldsymbol{\Gamma}$ for $X-34$.

\begin{tabular}{|c|c|c|c|c|c|c|c|c|c|}
\hline \multicolumn{2}{|c|}{ C-34 } & \multicolumn{4}{|c|}{$\mathrm{Si}-34$} & \multicolumn{4}{|c|}{ Ge-34 } \\
\hline \multirow{2}{*}{$\begin{array}{r}\omega_{q, \nu} \\
0\end{array}$} & \multirow{2}{*}{$\frac{\text { Sym. }}{T_{1 u}^{I R}}$} & \multirow{2}{*}{$\begin{array}{r}\omega_{q, \nu} \\
0\end{array}$} & \multirow{2}{*}{$\frac{\text { Sym. }}{T_{1 u}^{I R}}$} & \multicolumn{2}{|c|}{ Expt. (Refs. 40 and 41) } & \multirow{2}{*}{$\begin{array}{r}\omega_{q, \nu} \\
0\end{array}$} & \multirow{2}{*}{$\frac{\text { Sym. }}{T_{1 u}^{I R}}$} & \multicolumn{2}{|c|}{ Theo. (Ref. 34) } \\
\hline & & & & & & & & 0 & $T_{1 u}^{I R}$ \\
\hline 513 & $T_{2 g}^{R}$ & 119 & $T_{2 g}^{R}$ & $117 / 120$ & $T_{2 g}^{R}$ & 69 & $T_{2 g}^{R}$ & 54 & $T_{2 g}^{R}$ \\
\hline 538 & $E_{g}^{R}$ & 131 & $T_{1 g}$ & & & 76 & $T_{1 g}$ & 72 & $T_{1 g}$ \\
\hline 562 & $T_{1 g}^{g}$ & 133 & $E_{g}^{R}$ & $130 / 133$ & $E_{g}^{R}$ & 77 & $E_{g}^{R}$ & 74 & $E_{g}^{R}$ \\
\hline 641 & $T_{1 g}$ & 149 & $T_{2 u}$ & & & 86 & $T_{2 u}$ & 79 & $T_{1 u}^{I R}$ \\
\hline 657 & $T_{2 u}$ & 154 & $T_{1 g}$ & & & 87 & $T_{1 g}$ & 81 & $T_{2 u}$ \\
\hline 690 & $E_{u}$ & 163 & $E_{u}$ & & & 93 & $E_{u}$ & 81 & $T_{1 g}$ \\
\hline 721 & $T_{1 u}^{I R}$ & 171 & $T_{1 u}^{I R}$ & & & 95 & $T_{2 u}$ & 84 & $T_{2 g}^{R}$ \\
\hline 776 & $T_{2 u}$ & 174 & $T_{2 u}$ & & & 96 & $T_{1 u}^{I R}$ & 88 & $E_{u}$ \\
\hline 827 & $T_{2 g}^{R}$ & 177 & $T_{1 g}$ & & & 96 & $T_{2 g}^{R}$ & 90 & $T_{1 g}$ \\
\hline 847 & $T_{1 u}^{I R}$ & 180 & $T_{2 g}^{R}$ & $184 / 165$ & $T_{2 g}^{R}$ & 97 & $T_{1 g}^{2 g}$ & 91 & $T_{2 u}$ \\
\hline 863 & $T_{1 u}^{I R}$ & 181 & $A_{2 g}$ & & & 98 & $A_{2 g}$ & 93 & $A_{2 g}$ \\
\hline 869 & $T_{1 g}$ & 194 & $T_{1 u}^{I R}$ & & & 104 & $T_{1 u}^{I R}$ & 97 & $T_{1 u}^{I R}$ \\
\hline 874 & $A_{2 u}$ & 268 & $T_{1 u}^{I R}$ & & & 156 & $T_{1 u}^{I R}$ & 154 & $T_{1 u}^{I R}$ \\
\hline 894 & $A_{1 g}^{R}$ & 270 & $T_{2 g}^{R}$ & $271 / 272$ & $T_{2 g}^{R}$ & 159 & $T_{2 g}^{R}$ & 157 & $T_{2 g}^{R}$ \\
\hline 906 & $A_{2 g}$ & 283 & $A_{2 u}$ & & & 166 & $A_{2 u}$ & 162 & $A_{2 u}$ \\
\hline 913 & $T_{2 g}^{R}$ & 294 & $E_{u}$ & & & 173 & $E_{u}$ & 170 & $E_{u}$ \\
\hline 927 & $E_{u}$ & 318 & $A_{1 g}^{R}$ & $(316) / 320$ & $A_{1 g}^{R}$ & 187 & $A_{1 g}^{R}$ & 180 & $A_{1 g}^{R}$ \\
\hline 947 & $T_{2 g}^{R}$ & 326 & $T_{2 g}^{R}$ & $324 / 333$ & $T_{2 g}^{R}$ & 191 & $T_{2 g}^{R}$ & 187 & $T_{2 g}^{R}$ \\
\hline 958 & $E_{g}^{R}$ & 362 & $E_{g}^{R}$ & $360 / 362$ & $E_{g}^{R}$ & 211 & $E_{g}^{R}$ & 205 & $E_{g}^{R}$ \\
\hline 1003 & $T_{2 g}^{\delta}$ & 368 & $T_{2 u}^{s}$ & & & 216 & $T_{2 u}^{8}$ & 210 & $T_{2 u}^{8}$ \\
\hline 1020 & $A_{2 u}$ & 381 & $T_{1 u}^{I R}$ & & & 223 & $T_{1 u}^{I R}$ & 218 & $T_{1 u}^{I R}$ \\
\hline 1032 & $A_{1 g}^{R}$ & 397 & $A_{2 u}$ & & & 233 & $A_{1 g}^{R}$ & 225 & $A_{2 u}$ \\
\hline 1046 & $T_{1 u}^{I R}$ & 397 & $A_{1 g}^{R}$ & $387 / 383$ & $A_{1 g}^{R}$ & 234 & $A_{2 u}$ & 227 & $A_{1 g}^{R}$ \\
\hline 1056 & $E_{u}$ & 405 & $T_{2 g}^{R}$ & $401 / 404$ & $T_{2 g}^{R}$ & 237 & $T_{2 g}^{R}$ & 234 & $T_{2 g}^{R}$ \\
\hline 1076 & $T_{2 u}$ & 416 & $A_{2 u}$ & & & 244 & $A_{2 u}$ & 236 & $A_{2 u}$ \\
\hline 1094 & $T_{1 g}$ & 421 & $T_{1 g}$ & & & 246 & $T_{1 g}$ & 243 & $T_{1 g}$ \\
\hline 1107 & $A_{1 u}$ & 427 & $T_{1 u}^{I R}$ & & & 250 & $T_{1 u}^{I R}$ & 248 & $T_{1 u}^{I R}$ \\
\hline 1114 & $T_{1 u}^{I R}$ & 447 & $E_{u}$ & & & 265 & $E_{u}$ & 261 & $E_{u}$ \\
\hline 1137 & $E_{g}^{R}$ & 456 & $A_{1 g}^{R}$ & $454 /$ & $A_{1 g}^{R}$ & 271 & $T_{2 u}$ & 268 & $T_{2 u}$ \\
\hline 1145 & $T_{2 u}^{8}$ & 456 & $T_{2 u}$ & & & 272 & $A_{1 g}^{R}$ & 269 & $T_{1 u}^{I R}$ \\
\hline 1171 & $A_{2 u}$ & 460 & $T_{1 u}^{I R}$ & & & 273 & $T_{1 u}^{I R}$ & 270 & $A_{1 g}^{R}$ \\
\hline 1175 & $T_{2 g}^{R}$ & 462 & $E_{u}$ & & & 275 & $E_{u}$ & 271 & $E_{u}$ \\
\hline 1181 & $T_{2 u}$ & 463 & $E_{g}^{R}$ & $466 / 454$ & $E_{g}^{R}$ & 276 & $E_{g}^{R}$ & 273 & $E_{g}^{R}$ \\
\hline 1189 & $A_{1 g}^{R}$ & 468 & $T_{2 g}^{R}$ & $/ 467$ & $\begin{array}{l}g \\
T_{2 g}^{R}\end{array}$ & 279 & $T_{2 g}^{R}$ & 274 & $T_{2 g}^{R}$ \\
\hline 1198 & $T_{1 u}^{I R}$ & 473 & $T_{2 g}^{R g}$ & $472 / 471$ & $T_{2 g}^{R}$ & 282 & $T_{2 g}^{R}$ & 275 & $T_{1 u}^{I R}$ \\
\hline 1200 & $T_{2 g}^{R}$ & 475 & $T_{1 u}^{I R}$ & & & 283 & $T_{1 g}$ & 276 & $T_{2 g}^{R}$ \\
\hline 1204 & $E_{u}$ & 475 & $T_{1 g}$ & & & 284 & $T_{1 u}^{I R}$ & 279 & $T_{1 g}^{2 g}$ \\
\hline 1218 & $T_{1 u}^{I R}$ & 478 & $A_{1 u}$ & & & 285 & $A_{1 u}$ & 281 & $T_{2 g}^{R}$ \\
\hline 1227 & $T_{1 g}$ & 482 & $E_{g}^{R}$ & $480 /$ & $E_{g}^{R}$ & 287 & $E_{g}^{R}$ & 282 & $A_{1 u}$ \\
\hline 1245 & $E_{g}^{R}$ & 482 & $T_{2 u}^{g}$ & & & 289 & $T_{2 u}$ & 283 & $E_{g}^{R}$ \\
\hline 1250 & $T_{2 g}^{\stackrel{s}{R}}$ & 485 & $T_{2 g}^{R}$ & $488 / 490$ & $T_{2 g}^{R}$ & 290 & $T_{2 g}^{R}$ & 284 & $T_{2 u}^{g}$ \\
\hline
\end{tabular}

man scattering spectra, at $200 \mathrm{~cm}^{-1}$ the relative intensity of the $T_{2 g}$ mode is correctly reproduced in comparison to other peaks but at low frequency we have two peaks with the same intensity.
The carbon spectra are significantly different from those of silicon or germanium networks: for C-34 only one mode $A_{1 g}$ should present a significant active frequency, in parallel measurement. In contrast a perpendicular mea- 
TABLE VI. Frequencies (in $\mathrm{cm}^{-1}$ ) and symmetries of the vibrational modes in $\boldsymbol{\Gamma}$ for $X-46$ systems.

\begin{tabular}{|c|c|c|c|c|c|c|c|c|c|}
\hline \multicolumn{2}{|c|}{ C-46 } & \multicolumn{4}{|c|}{$\mathrm{Si}-46$} & \multicolumn{4}{|c|}{$\mathrm{Ge}-46$} \\
\hline \multirow{2}{*}{$\begin{array}{r}\omega_{q, \nu} \\
0\end{array}$} & \multirow{2}{*}{$\frac{\text { Sym. }}{T_{1 u}^{I R}}$} & \multirow{2}{*}{$\frac{\omega_{q, \nu}}{0}$} & \multirow{2}{*}{$\frac{\text { Sym. }}{T_{1 u}^{I R}}$} & \multicolumn{2}{|c|}{ Theo. (Ref. 42) } & \multirow{2}{*}{$\frac{\omega_{q, \nu}}{0}$} & \multirow{2}{*}{$\frac{\text { Sym. }}{T_{1 u}^{I R}}$} & \multicolumn{2}{|c|}{ Theo. (Ref. 34) } \\
\hline & & & & 0 & $T_{1 u}^{I R}$ & & & 0 & $T_{1 u}^{I R}$ \\
\hline 457 & $T_{2 g}^{R}$ & 114 & $T_{2 g}^{R}$ & 114 & $T_{2 g}^{R}$ & 65 & $T_{2 g}^{R}$ & 62 & $T_{2 g}^{R}$ \\
\hline 505 & $T_{1 g}$ & 120 & $T_{1 g}$ & 126 & $T_{1 g}$ & 70 & $T_{1 g}$ & 63 & $T_{1 g}$ \\
\hline 576 & $T_{2 u}$ & 129 & $A_{2 u}$ & 143 & $T_{2 u}$ & 76 & $A_{2 u}$ & 67 & $E_{g}^{R}$ \\
\hline 588 & $E_{g}^{R}$ & 140 & $T_{1 g}$ & 144 & $T_{1 u}^{I R}$ & 79 & $T_{1 g}$ & 69 & $T_{1 u}^{I R}$ \\
\hline 591 & $T_{1 u}^{I R}$ & 140 & $T_{2 u}$ & 157 & $T_{1 g}$ & 81 & $T_{2 u}$ & 71 & $T_{2 u}$ \\
\hline 623 & $A_{2 u}$ & 143 & $T_{1 u}^{I R}$ & 164 & $T_{1 u}^{I R}$ & 82 & $T_{1 u}^{I R}$ & 74 & $T_{1 g}$ \\
\hline 623 & $T_{1 g}$ & 147 & $E_{g}^{R}$ & 172 & $E_{g}^{R}$ & 82 & $E_{g}^{R}$ & 77 & $T_{2 g}^{R}$ \\
\hline 637 & $A_{2 g}$ & 153 & $E_{u}$ & 182 & $E_{u}$ & 86 & $E_{u}$ & 78 & $A_{2 u}$ \\
\hline 682 & $T_{2 u}$ & 166 & $T_{1 g}$ & 194 & $T_{2 u}$ & 93 & $T_{1 g}$ & 79 & $A_{2 g}$ \\
\hline 695 & $E_{u}$ & 169 & $T_{2 u}$ & 197 & $T_{1 g}$ & 93 & $A_{2 g}$ & 82 & $E_{u}$ \\
\hline 716 & $E_{g}^{R}$ & 170 & $T_{2 g}^{R}$ & 198 & $T_{2 g}^{R}$ & 94 & $T_{2 g}^{R}$ & 83 & $T_{1 g}$ \\
\hline 732 & $T_{1 g}$ & 173 & $T_{1 u}^{I R}$ & 208 & $T_{1 u}^{I R}$ & 94 & $T_{2 u}$ & 89 & $T_{1 u}^{I R}$ \\
\hline 752 & $T_{2 g}^{R}$ & 175 & $A_{2 g}$ & 220 & $E_{u}$ & 96 & $T_{2 g}^{R}$ & 89 & $T_{2 g}^{R}$ \\
\hline 781 & $T_{1 u}^{I R}$ & 178 & $T_{2 g}^{R}$ & 224 & $E_{g}^{R}$ & 96 & $T_{1 g}$ & 89 & $T_{1 \mathrm{~g}}$ \\
\hline 818 & $T_{2 u}$ & 178 & $E_{u}$ & 225 & $A_{2 g}$ & 96 & $T_{1 u}^{I R}$ & 89 & $T_{2 u}$ \\
\hline 824 & $E_{g}^{R}$ & 180 & $T_{1 g}$ & 236 & $T_{2 g}^{R}$ & 97 & $E_{u}$ & 91 & $E_{u}$ \\
\hline 851 & $E_{u}$ & 194 & $T_{1 u}^{I R}$ & 237 & $T_{1 g}$ & 103 & $T_{1 u}^{I R}$ & 93 & $T_{1 u}^{I R}$ \\
\hline 879 & $T_{2 g}^{R}$ & 223 & $E_{g}^{R}$ & 240 & $A_{2 g}$ & 131 & $E_{g}^{R}$ & 131 & $E_{g}^{R}$ \\
\hline 878 & $A_{2 g}$ & 239 & $A_{2 g}$ & 246 & $T_{2 u}$ & 138 & $A_{2 g}$ & 138 & $T_{2 u}$ \\
\hline 882 & $T_{1 u}^{I R}$ & 247 & $T_{2 u}$ & 250 & $T_{1 u}^{I R}$ & 142 & $T_{2 u}$ & 139 & $A_{2 g}$ \\
\hline 909 & $T_{1 g}$ & 301 & $T_{1 u}^{I R}$ & 307 & $E_{g}$ & 176 & $E_{g}^{R}$ & 170 & $E_{g}^{R}$ \\
\hline 920 & $T_{2 g}^{R}$ & 303 & $E_{g}^{R}$ & 309 & $T_{1 u}^{I R}$ & 177 & $T_{1 u}^{I R}$ & 172 & $T_{1 u}^{G R}$ \\
\hline 946 & $T_{1 u}^{I R}$ & 305 & $T_{2 g}^{\mathcal{K}}$ & 314 & $T_{2 g}^{R}$ & 179 & $T_{2 g}^{R}$ & 176 & $T_{2 g}^{R}$ \\
\hline 948 & $A_{1 g}^{R}$ & 325 & $T_{2 u}$ & 332 & $T_{2 u}$ & 190 & $T_{2 u}$ & 186 & $T_{2 u}$ \\
\hline 955 & $A_{2 g}$ & 326 & $A_{1 u}$ & 345 & $A_{1 g}^{R}$ & 193 & $A_{1 u}$ & 189 & $A_{1 g}^{R}$ \\
\hline 992 & $T_{2 u}$ & 334 & $A_{1 g}^{R}$ & 349 & $A_{1 u}$ & 195 & $A_{1 g}^{R}$ & 190 & $A_{1 u}$ \\
\hline 1007 & $T_{1 u}^{I R}$ & 355 & $T_{1 g}$ & 372 & $T_{1 g}$ & 209 & $T_{1 g}$ & 206 & $T_{1 g}$ \\
\hline 1015 & $A_{1 u}$ & 370 & $T_{2 u}$ & 390 & $T_{2 u}$ & 217 & $T_{2 u}$ & 212 & $T_{2 u}$ \\
\hline 1025 & $T_{2 u}$ & 375 & $T_{1 u}^{I R}$ & 393 & $T_{1 u}^{I R}$ & 220 & $T_{1 u}^{I R}$ & 215 & $T_{1 u}^{I R}$ \\
\hline 1033 & $T_{1 g}$ & 383 & $A_{2 g}$ & 403 & $A_{2 g}$ & 223 & $A_{2 g}$ & 218 & $A_{2 g}$ \\
\hline 1055 & $T_{1 u}^{I R}$ & 384 & $A_{2 u}$ & 409 & $A_{2 u}$ & 226 & $A_{2 u}$ & 223 & $A_{2 u}$ \\
\hline 1058 & $A_{1 g}^{R}$ & 408 & $T_{2 g}^{R}$ & 423 & $E_{g}^{R}$ & 240 & $T_{2 g}^{R}$ & 235 & $T_{1 u}^{I R}$ \\
\hline 1062 & $E_{g}^{R}$ & 414 & $E_{g}^{R}$ & 429 & $T_{2 u}$ & 242 & $T_{1 u}^{I R}$ & 235 & $T_{2 g}^{R}$ \\
\hline 1075 & $T_{1 g}$ & 418 & $T_{1 u}^{I R}$ & 431 & $T_{2 g}^{R}$ & 242 & $E_{g}^{R}$ & 238 & $E_{g}^{R}$ \\
\hline 1082 & $T_{2 u}$ & 418 & $T_{1 g}$ & 441 & $T_{1 g}$ & 245 & $T_{1 g}$ & 240 & $T_{1 g}$ \\
\hline 1088 & $T_{2 g}^{R}$ & 432 & $A_{1 g}^{R}$ & 445 & $A_{1 g}^{R}$ & 251 & $A_{1 g}^{R}$ & 247 & $A_{1 g}^{R}$ \\
\hline 1088 & $T_{1 u}^{I R}$ & 442 & $T_{2 u}$ & 467 & $T_{2 u}$ & 260 & $T_{2 u}$ & 258 & $T_{2 u}$ \\
\hline 1092 & $E_{g}^{R}$ & 450 & $T_{1 u}^{I R}$ & 483 & $T_{1 u}^{I R}$ & 268 & $T_{1 u}^{I R}$ & 263 & $T_{1 u}^{I R}$ \\
\hline 1129 & $A_{2 u}$ & 459 & $E_{g}^{R}$ & 484 & $E_{g}^{R}$ & 272 & $E_{g}^{R}$ & 268 & $E_{g}^{R}$ \\
\hline 1140 & $T_{2 u}$ & 461 & $T_{2 u}$ & 489 & $T_{2 u}$ & 274 & $T_{2 u}$ & 271 & $T_{2 u}$ \\
\hline 1143 & $E_{u}$ & 462 & $E_{u}$ & 492 & $T_{1 u}^{I R}$ & 275 & $E_{u}$ & 272 & $T_{1 g}$ \\
\hline 1152 & $A_{2 g}$ & 465 & $T_{1 u}^{I R}$ & 493 & $A_{2 g}$ & 277 & $T_{1 u}^{I R}$ & 272 & $T_{2 g}^{R}$ \\
\hline 1158 & $T_{2 g}^{R}$ & 466 & $T_{2 g}^{R}$ & 495 & $T_{2 u}$ & 277 & $E_{u}$ & 272 & $T_{1 u}^{I R}$ \\
\hline 1167 & $T_{1 u}^{I R}$ & 467 & $T_{2 u}$ & 495 & $T_{2 u}$ & 277 & $T_{2 g}^{R}$ & 273 & $T_{2 u}$ \\
\hline 1170 & $E_{g}^{R}$ & 467 & $E_{u}$ & 498 & $E_{u}$ & 278 & $T_{1 u}^{I R}$ & 273 & $E_{u}$ \\
\hline 1192 & $A_{1 u}$ & 468 & $T_{1 g}$ & 498 & $E_{g}$ & 278 & $T_{2 u}$ & 275 & $T_{1 u}^{I R}$ \\
\hline
\end{tabular}


TABLE VI. (Continued.)

\begin{tabular}{|c|c|c|c|c|c|c|c|c|c|}
\hline \multicolumn{2}{|c|}{ C-46 } & \multicolumn{4}{|c|}{ Si-46 } & \multicolumn{4}{|c|}{ Ge-46 } \\
\hline \multirow{2}{*}{$\frac{\omega_{q, \nu}}{1192}$} & \multirow{2}{*}{$\frac{\text { Sym. }}{T_{1 g}}$} & \multirow{2}{*}{$\frac{\omega_{q, \nu}}{468}$} & \multirow{2}{*}{$\frac{\text { Sym. }}{T_{2 g}^{R}}$} & \multicolumn{2}{|c|}{ Theo. (Ref. 42) } & \multirow{2}{*}{$\frac{\omega_{q, \nu}}{278}$} & \multirow{2}{*}{$\frac{\text { Sym. }}{T_{1 g}}$} & \multicolumn{2}{|c|}{ Theo. (Ref. 34) } \\
\hline & & & & 498 & $T_{2 g}^{R}$ & & & 275 & $E_{u}$ \\
\hline 1198 & $E_{u}$ & 469 & $T_{1 u}^{R}$ & 498 & $T_{1 g}$ & 279 & $T_{2 g}^{R}$ & 275 & $E_{g}^{R}$ \\
\hline 1198 & $T_{2 u}$ & 470 & $E_{g}^{R}$ & 500 & $T_{1 u}^{I R}$ & 279 & $E_{g}^{R}$ & 276 & $T_{2 g}^{g}$ \\
\hline 1201 & $T_{2 g}^{R}$ & 470 & $A_{1 u}^{8}$ & 504 & $E_{u}$ & 279 & $A_{1 u}$ & 277 & $A_{1 u}^{2 g}$ \\
\hline 1202 & $T_{1 u}^{I R}$ & 471 & $T_{1 g}$ & 504 & $T_{1 g}$ & 281 & $T_{1 g}$ & 278 & $T_{1 g}$ \\
\hline 1220 & $T_{1 g}$ & 474 & $T_{2 g}^{R}$ & 505 & $T_{2 g}^{R}$ & 281 & $T_{2 g}^{R}$ & 278 & $T_{2 g}^{R}$ \\
\hline 1223 & $E_{g}^{R}$ & 479 & $T_{2 u}$ & 511 & $A_{1 g}^{R}$ & 284 & $E_{g}^{R}$ & 279 & $E_{g}^{R}$ \\
\hline 1229 & $T_{2 g}^{\bar{K}}$ & 480 & $E_{g}^{R}$ & 512 & $A_{1 u}$ & 284 & $A_{2 g}^{g}$ & 279 & $A_{2 g}^{g}$ \\
\hline 1260 & $T_{2 u}$ & 481 & $A_{2 g}$ & 515 & $T_{2 g}^{R}$ & 285 & $T_{2 u}$ & 282 & $T_{2 u}$ \\
\hline 1260 & $A_{1 g}^{R}$ & 481 & $A_{1 g}^{R}$ & 516 & $T_{2 u}$ & 286 & $A_{1 g}^{R}$ & 282 & $A_{1 g}^{R}$ \\
\hline
\end{tabular}

surement must show more signals (four groups of peaks). For C-46, we obtain a spectrum with six equivalent peaks.

\section{Infrared spectra}

We have calculated the infrared spectra from dynamical matrix, we have plotted it on the Fig. 5. Our results on sili-

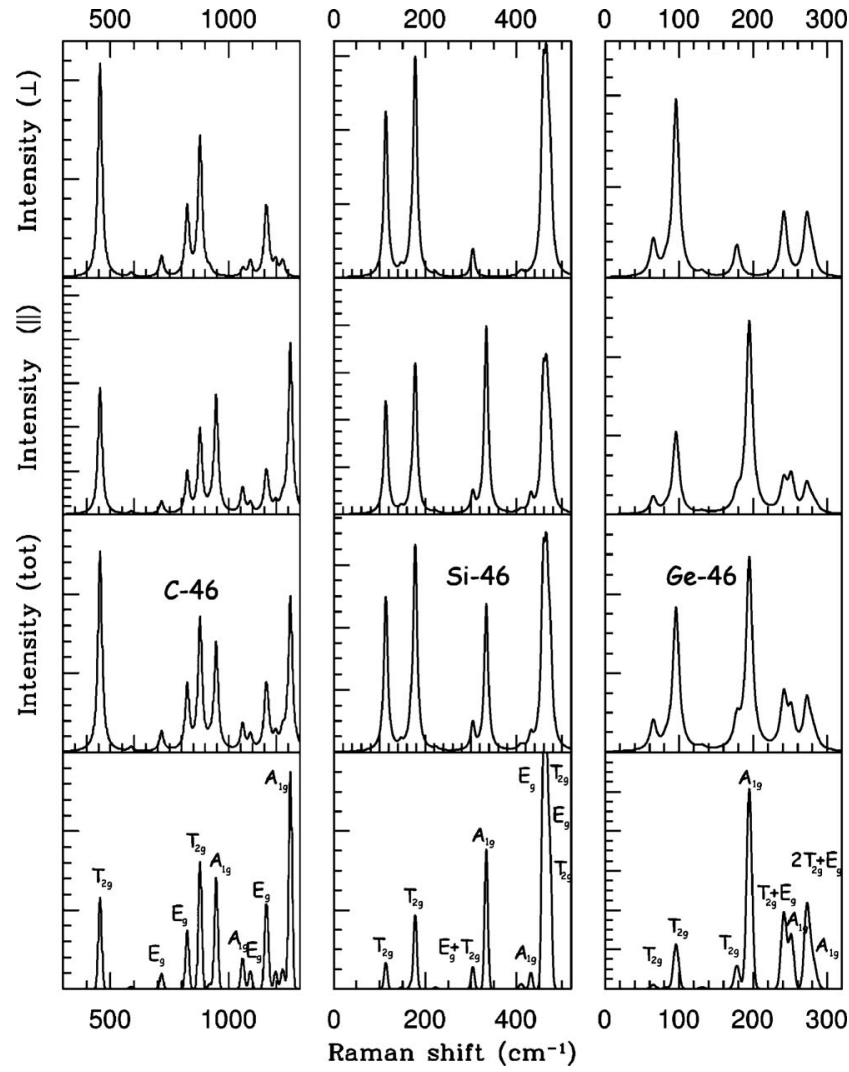

FIG. 3. Raman spectra of $X-46$ (from left to right: C, $\mathrm{Si}$, and $\mathrm{Ge}$ ) clathrates: from top to bottom figures: perpendicular, parallel, total Raman intensities, and total cross section convoluted with a uniform Gaussian broadening having $10-5 \mathrm{~cm}^{-1}$ width. We have used a damping constant $\Gamma_{i}=10 \mathrm{~cm}^{-1}$ for all modes. Peaks are labeled in function to their symmetries. con clathrates are in agreement with those published by Li $e t$ $a l .,{ }^{51}$ in spite of that the frequencies found by these authors are slightly shifted from ours. The infrared spectra are less sensitive to the element than Raman spectra. The spectra depend on the dynamical properties, which results from a strong similarity between systems.

\section{Polarizability and dielectric constants}

Finally, we report the polarizability $(\alpha)$ and the permittivity $\left(\epsilon_{\infty}\right)$ in Table VII. Quantitatively, dielectric constants are
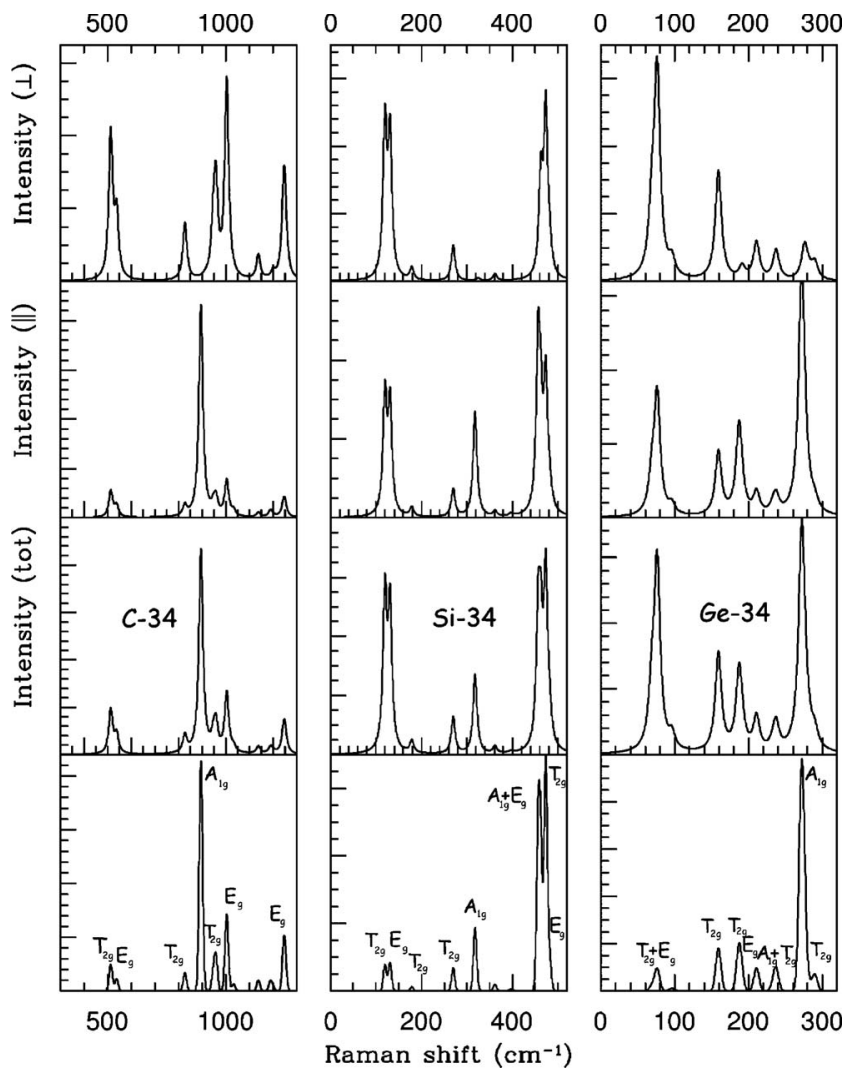

FIG. 4. Raman spectra of $X-34$ (from left to right: C, Si, and Ge) clathrates, see Fig. 3 for labels. 

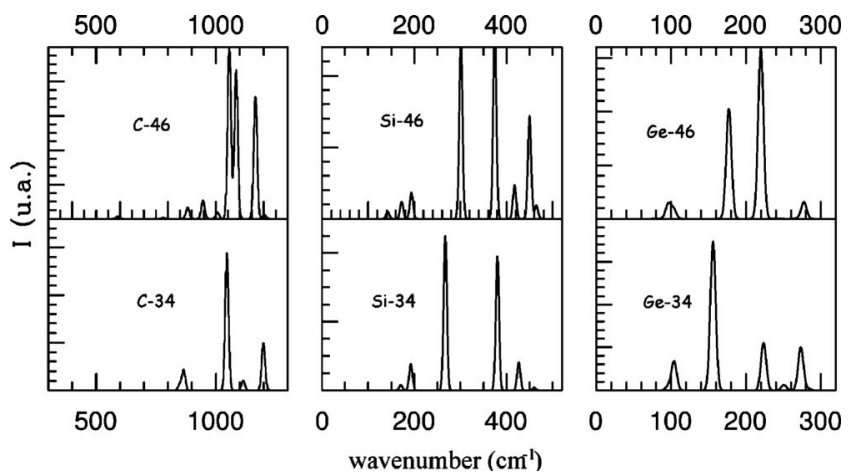

FIG. 5. Infrared spectra of $X-46$ and $X-34$ (from left to right: C, $\mathrm{Si}$, and $\mathrm{Ge}$ ) clathrates.

in agreement with the values found by Blase ${ }^{17}$ within a GW approximation. The polarizability is lighter greater in clathrates than in diamond, and the Born effective charge is small (less $<0.1$ for Born charges) as in diamond phases, which accounts for the lack of LO-TO splitting.

\section{B. Thermodynamical properties}

The thermodynamical properties of clathrates and diamonds under pressure and temperature have been investigated from the interatomic constants. To compute these properties, two approaches for comparison were performed: the static approximation and the quasiharmonic approximation (QHA). Only carbon systems have been studied within the framework of the QHA.

\section{Static approximation}

This approximation consists of a calculation of the vibrational free energy $\left(F_{v i b}\right)$ at the optimized lattice parameter,
TABLE VII. Dielectric constants $\left(\epsilon_{\infty}\right)$ and polarizabilities $\overleftrightarrow{\alpha}$ $=\alpha \stackrel{\leftrightarrow}{I}$ (in $\AA^{3} /$ atom).

\begin{tabular}{lcc}
\hline \hline & $\epsilon_{\infty}$ & $\alpha$ \\
\hline $\mathrm{C}-2$ & $5.7\left(5.5^{\mathrm{a}}\right)$ & 0.84 \\
$\mathrm{C}-34$ & $4.8\left(4.7^{\mathrm{b}}\right)$ & 0.86 \\
$\mathrm{C}-46$ & 4.9 & 0.87 \\
$\mathrm{Si}-2$ & $13.6\left(11.7^{\mathrm{a}}\right)$ & 3.73 \\
$\mathrm{Si}-34$ & $10.0\left(9.8^{\mathrm{b}}\right)$ & 4.06 \\
$\mathrm{Si}-46$ & 10.4 & 4.03 \\
$\mathrm{Ge}-2$ & $18.3\left(15.8^{\mathrm{a}}\right)$ & 4.40 \\
$\mathrm{Ge}-34$ & 12.4 & 4.67 \\
$\mathrm{Ge}-46$ & 12.8 & 4.65 \\
\hline
\end{tabular}

axperiment (Ref. 31).

bTheory GW calculations, Ref. 17.

where the effect of the temperature on the lattice parameter is neglected. The Helmholtz free energy, for nonmetallic systems, is then given by

$$
F(T)=U_{o}+F_{\mathrm{vib}}(T),
$$

where $U_{o}$ is the internal energy of the cell at the optimized volume and

$$
F_{\text {vib }}(T)=k_{B} T \sum_{\mathbf{q}, \nu} \ln \left[2 \sinh \left(\xi_{\mathbf{q}, \nu}\right)\right]
$$

the vibrational free energy $\left(\xi_{\mathbf{q}, \nu}=\hbar \omega_{\mathbf{q}, \nu} / 2 k_{B} T\right)$. The summation on the vibration frequencies has been performed on 20 $\times 20 \times 20$ tetrahedron grids. The heat capacity and the entropy have been evaluated following the relations:
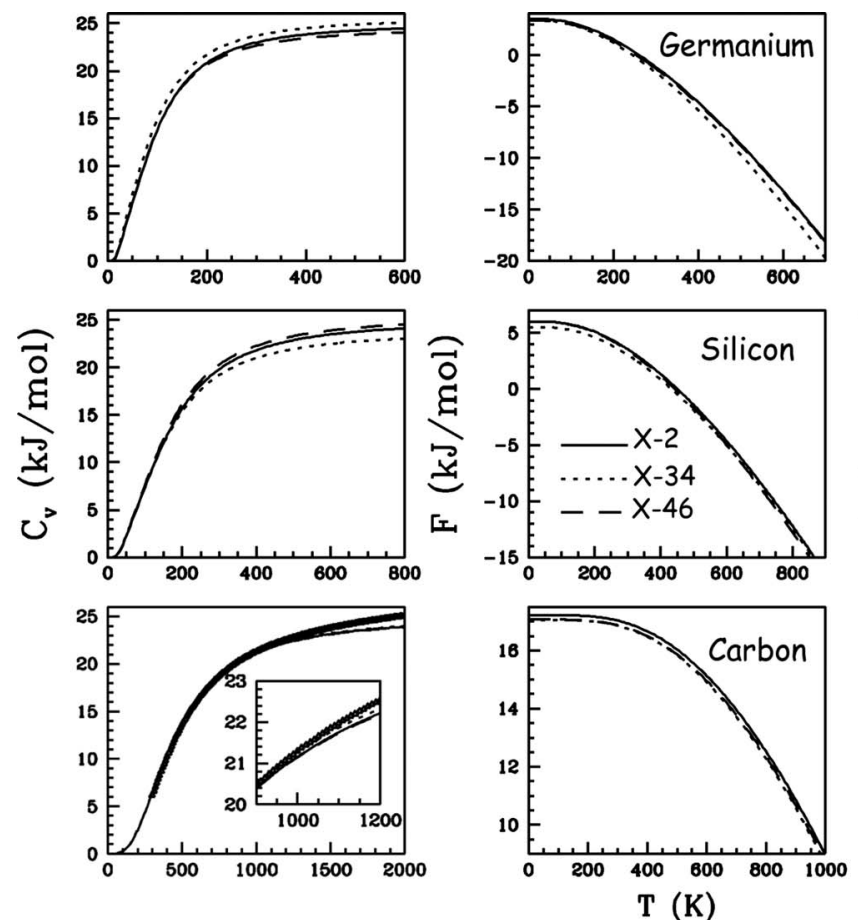
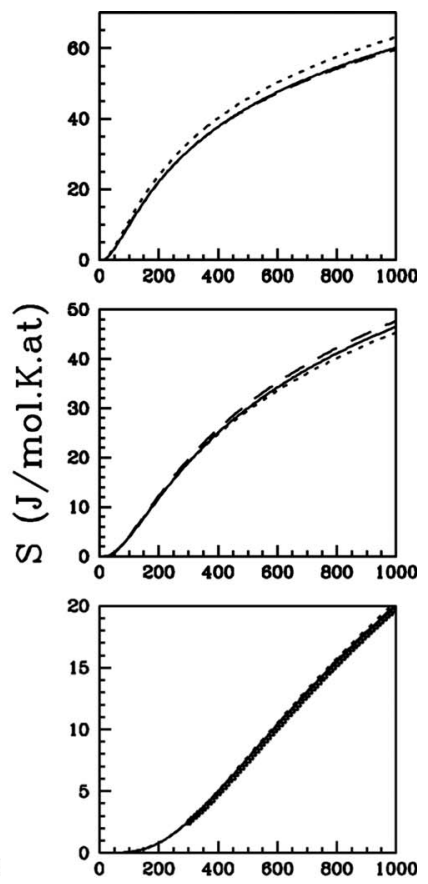

FIG. 6. Variation in the heat capacity $\left(C_{v}\right)$, free energy $(F)$, and entropy $(S)$ in clathrates and diamond in the static approximation, from bottom to top: carbon, silicon, and germanium systems. Dots represent the experimental points $\left(C_{p}\right.$, and $\left.S\right)$ of carbon diamond (Ref. 52). 

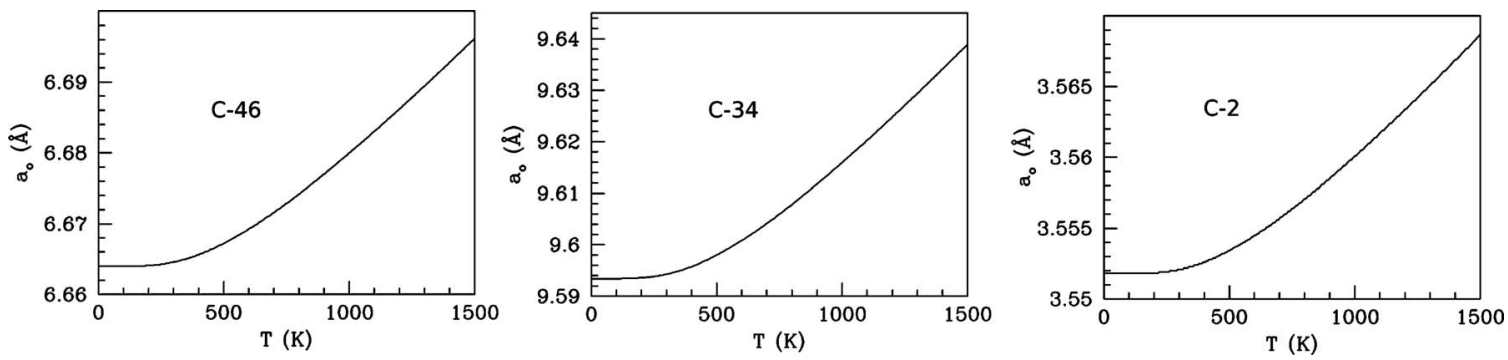

FIG. 7. Variation in the lattice parameter with respect to the temperature at $0 \mathrm{GPa}$.

$$
\begin{gathered}
C_{v}(T)=-T \frac{d^{2} F_{\mathrm{vib}}}{d T^{2}}=-k_{B} \sum_{\mathbf{q}, \nu}\left[\frac{\xi_{\mathbf{q}, \nu}}{\sinh \left(\xi_{\mathbf{q}, \nu}\right)}\right]^{2}, \\
S(T)=S_{\mathrm{vib}}(T)=-\frac{d F_{\mathrm{vib}}}{d T} .
\end{gathered}
$$

$S, C_{v}$, and $F$ are plotted Fig. 6 for $\mathrm{C}, \mathrm{Si}$, and Ge systems, and compared with experimental data $\left(C_{p}\right.$ and $\left.S\right)$ for carbon diamond. ${ }^{52}$ Until around $800 \mathrm{~K}$, the static approximation reproduces accurately the heat capacity and entropy of carbon diamond. The results, reported for $\mathrm{Si} / \mathrm{Ge}$ clathrates are in good agreement with those published by Biswas ${ }^{53}$ and Tang. ${ }^{41}$ In conclusion, according to the static approximation, diamond and clathrates behavior are, for a given element, slightly different.

\section{2. $Q H A$}

If the anharmonic effects are neglected at the level of the QHA, the thermal expansion is restored by introducing the (anharmonic) effect of the temperature and the pressure on lattice parameters and thermodynamic properties in the Eq. (3). Isothermal fourth-order Birch-Murnaghan binding energy relation has been used to fit the free energy

$$
F(a, T)=F_{o}+\frac{9}{8} B_{o} V_{o} u^{2}+A u^{3}+C u^{4}+\mathcal{O}\left[u^{5}\right],
$$

where $u=\left(V_{o} / V\right)^{2 / 3}-1$. For a given temperature, the free energy has been fitted by a spline-type approximation. The pressure is then deduced from the following relation:

$$
P(T, V)=-\left.\frac{\partial F(T, V)}{\partial V}\right|_{T}
$$
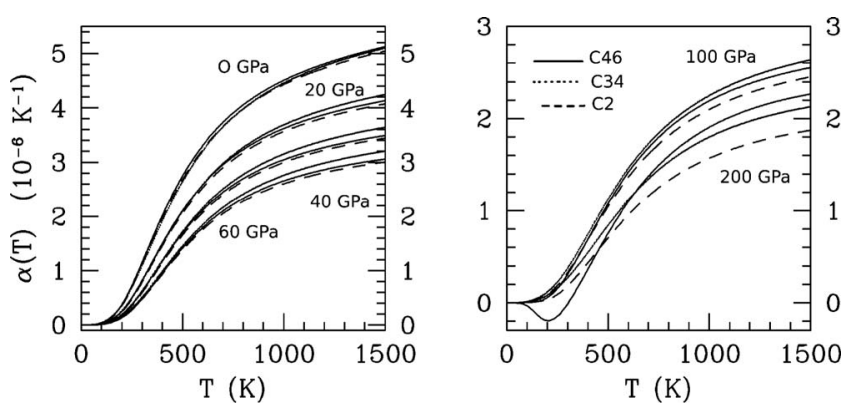

FIG. 8. Variation in the linear thermal $\alpha(T)$ for C-2 (dots), C-46 (line), and C-34 (dashed lines) with the temperature for different pressures: $0<P<60 \mathrm{GPa}$ (left), and 100, $200 \mathrm{GPa}$ (right).
$V(T, P)$ is thus interpolated from $P(T, V)$, the Gibbs energy $G(T, P)$ is computed as the function of the pressure and temperature. The thermal dilatation coefficient and the Grüneisen parameter are deduced from

$$
\begin{gathered}
\alpha_{v}(T, P)=\left.\frac{1}{V} \frac{\partial V}{\partial T}\right|_{P}, \\
\gamma(T, P)=V B_{o} \alpha / C_{v}
\end{gathered}
$$

while the heat capacity $C_{p}$ and $C_{v}=\partial U / \partial T$ are linked by

$$
C_{p}(T, P)=C_{v}+\alpha_{v}^{2} B_{o} V T .
$$

To calculate $\mathrm{F}_{v i b}$ we have restricted the summation on $q$ points at the only $\boldsymbol{\Gamma}$ point for carbon systems. The temperature dependence of the lattice parameter and thermal expansion, respectively, are plotted in Figs. 7 and 8 up to $1500 \mathrm{~K}$ at $0 \mathrm{GPa}$. The $P-V-T$ relationship of diamond and clathrates is reported in Fig. 9 for two temperatures.

The pressure and temperature behavior of $\mathrm{C}_{p}, \mathrm{C}_{v}$, and $\alpha$ of the three systems are very similar (Fig. 10). Differences occur at high pressure (up to $150 \mathrm{GPa}$ ), where the lattice parameter of C-46 is reduced contrary to C-34 and C-2, for which the related reduction occurs at higher pressure (around $700 \mathrm{GPa}){ }^{54}$ The Grüneisen parameters of Raman modes $\left[\gamma_{i}=-\frac{\partial \ln \left(\omega_{i}\right)}{\partial \ln (\mathrm{V})}\right]$ have been calculated and reported in Table VIII. For carbon diamond one found $\gamma=1.01$ for the Raman active mode at $1307 \mathrm{~cm}^{-1}$, in agreement with experimental measurement. ${ }^{55}$ Contrary to $\mathrm{Si}-34,{ }^{41}$ all Grüneisen parameters of carbon clathrates are positive. The Debye temperatures $\left(\theta_{D}\right)$, of clathrates, calculated and reported in Table IX, are $10 \%$ smaller than the diamond temperature.

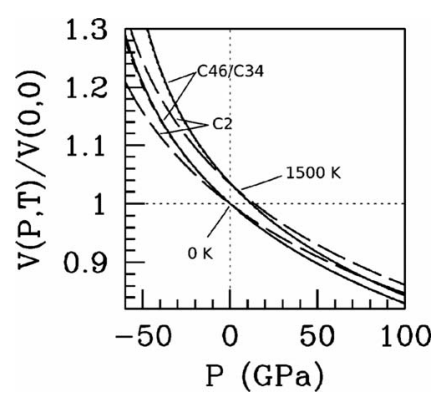

FIG. 9. Calculated $P-V-T$ relationship of diamond and clathrates for two temperatures $(0 \mathrm{~K}$ and $1500 \mathrm{~K})$ taken as reference the volume at $0 \mathrm{~K}$ and $0 \mathrm{GPa}$. 


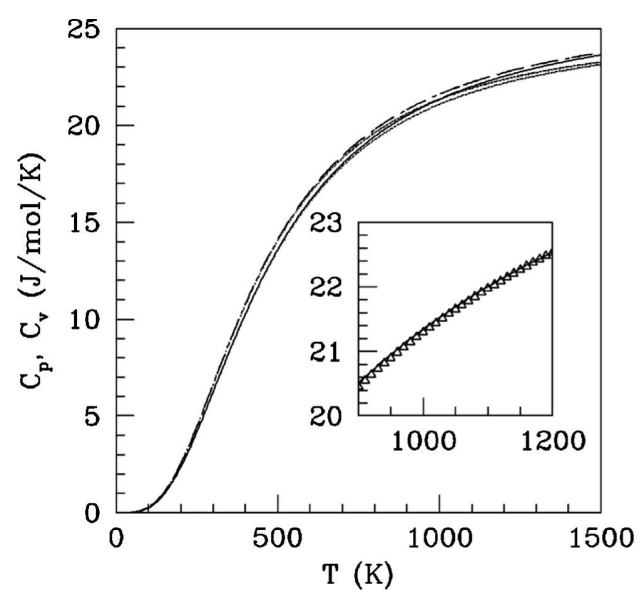

\section{C. $(P, T)$ Phase diagram}

We have investigated the phase diagram of carbon phases, by assuming the same Gibbs energies for carbon diamond with carbon clathrates, the temperature of the transition for a pressure. Figure 11 shows the phase diagram from 0 to $1500 \mathrm{~K}$. At $0 \mathrm{~K}$,the transition pressures $P_{t}$ are -21 and $-28 \mathrm{GPa}$ for $\mathrm{C}-46$ and $\mathrm{C}-34$, respectively. These negative pressures agree with those calculated by Perottoni et al. ${ }^{13}$

TABLE VIII. Calculated Raman frequencies $\left(\omega_{i}\right)$ and Grüneisen parameters $\left[\gamma_{i}=-\partial \ln \left(\omega_{i}\right) / \partial \ln (V)\right]$ for C-34 and C-46.

\begin{tabular}{|c|c|c|c|}
\hline \multicolumn{2}{|c|}{ C-34 } & \multicolumn{2}{|c|}{ C-46 } \\
\hline $\begin{array}{c}\omega_{i} \\
\left(\mathrm{~cm}^{-1}\right)\end{array}$ & $\gamma_{i}$ & $\begin{array}{c}\omega_{i} \\
\left(\mathrm{~cm}^{-1}\right)\end{array}$ & $\gamma_{i}$ \\
\hline \multicolumn{4}{|c|}{$E_{g}$} \\
\hline 538 & 0.52 & 588 & 0.76 \\
\hline 958 & 1.14 & 716 & 1.01 \\
\hline 1137 & 1.24 & 824 & 1.49 \\
\hline \multirow[t]{4}{*}{1245} & 1.17 & 1062 & 1.44 \\
\hline & & 1092 & 1.62 \\
\hline & & 1170 & 1.22 \\
\hline & & 1223 & 0.88 \\
\hline \multicolumn{4}{|c|}{$A_{1 g}$} \\
\hline 894 & 1.59 & 948 & 1.18 \\
\hline 1032 & 1.11 & 1058 & 1.75 \\
\hline 1189 & 0.80 & 1260 & 1.02 \\
\hline \multicolumn{4}{|c|}{$T_{2 g}$} \\
\hline 513 & 0.26 & 457 & 0.48 \\
\hline 827 & 1.19 & 752 & 0.60 \\
\hline 913 & 0.35 & 879 & 0.49 \\
\hline 947 & 1.22 & 920 & 0.93 \\
\hline 1003 & 1.32 & 1088 & 1.05 \\
\hline 1175 & 1.36 & 1158 & 1.20 \\
\hline 1200 & 0.96 & 1201 & 0.97 \\
\hline 1250 & 0.97 & 1229 & 0.99 \\
\hline
\end{tabular}

FIG. 10. Variation in the heat capacity $\left(C_{v}\right.$ and $\left.C_{p}\right)$ and Grüneisen parameter $[\gamma$ $=\partial \ln (\Theta) / \partial \ln (V)]$ at $0 \mathrm{GPa}$. We have enlarged around $1000 \mathrm{~K}$ experimental and theoretical $C_{p}$ of carbon diamond (dots represent experimental data (Ref. 52), dashed lines simulations). (using a Hartree-Fock approximation), and Rey et al. ${ }^{16}$

Gibbs' phase rule for one component system shows that two phases coexist along a curve in the $T, P$ plane, where the Clausius-Clapeyron equation $\left(d P_{t} / d T=\Delta S / \Delta V\right)$ is the coexistence curve. We found $d P_{t} / d T>0$ for C-46/C2 and C-34/ $\mathrm{C} 2$. Negative pressure values are expected in those kinds of transitions implying expanded phases compared to diamond. In absolute values, the transition pressures are found much larger than those calculated for Si-2/Si-46 (-6 GPa) and Ge2/Ge-46 (-2.4 GPa).

\section{Elasticity}

The elastic constants $\left(C_{i j}\right)$ have been derived from vibrational acoustic dispersion slopes presented above. In cubic systems, the elastic tensor can be described by three independent elastic constants (see Ref. 31): namely, $C_{11}, C_{12}$, and $C_{44}$. The slope of the acoustic branches along the [100] direction are related to $C_{11}$ and $C_{44}$

$$
v_{l}^{[100]}=\sqrt{\frac{C_{11}}{\rho}}, v_{t}^{[100]}=\sqrt{\frac{C_{44}}{\rho}},
$$

where $v_{l}^{[100]}$ and $v_{t}^{[100]}$ are, respectively, the longitudinal and transverse velocities of the sound and $\rho$ the density. For $C_{12}$, we use the [111] direction: $v_{l}^{[111]}=\sqrt{\frac{C_{11}+2 C_{12}+4 C_{44}}{3 \rho}}$.

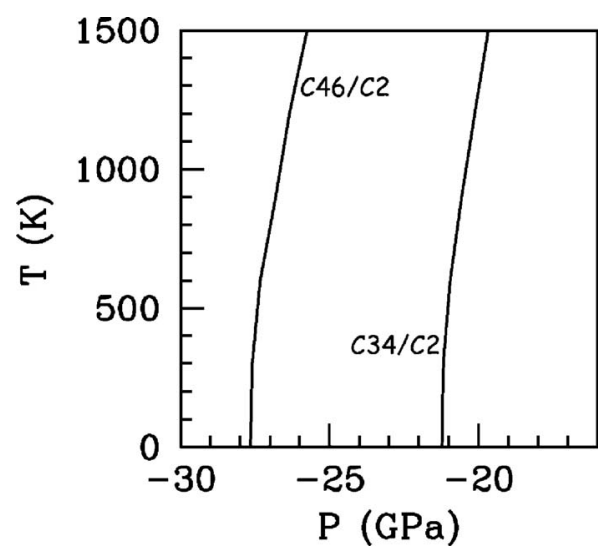

FIG. 11. Equilibrium phase diagram $(P, T)$ of carbon diamond and clathrates. 
TABLE IX. The densities (in $\mathrm{kg} / \mathrm{m}^{3}$ ), the elastic constants (in GPa), the bulk modulus (in $\mathrm{GPa}$ ), the factors of anisotropy $C_{a}$, the shears moduli $G$ (in GPa), the Young modulus $Y$ along the [100] direction (in GPa), the Poisson ratio $\nu$, the Young modulus along the [111] direction (in GPa), and the Debye temperatures (in K) for each system.

\begin{tabular}{|c|c|c|c|c|c|c|c|c|c|c|c|}
\hline & $\rho$ & $C_{11}$ & $C_{12}$ & $C_{44}$ & $B_{o}$ & $C_{a}$ & $G$ & $Y_{100}$ & $\nu$ & $Y_{111}$ & $\theta_{D}$ \\
\hline $\mathrm{C}-2$ & 3593 & $1092\left(1076^{a}\right)$ & $135\left(125^{\mathrm{a}}\right)$ & $585\left(576^{a}\right)$ & 458 & 0.81 & 475 & 1060 & 0.11 & 1231 & 2230 \\
\hline C-34 & 3098 & 966 & 87 & 437 & 381 & 0.99 & 439 & 952 & 0.08 & 947 & 2083 \\
\hline C-46 & 3129 & $935\left(960^{\mathrm{b}}\right)$ & $127\left(117^{\mathrm{b}}\right)$ & $462\left(472^{b}\right)$ & 396 & 0.87 & 403 & 903 & 0.11 & 996 & 1996 \\
\hline $\mathrm{Si}-2$ & 2381 & $167\left(166^{\mathrm{a}}\right)$ & $63\left(64^{\mathrm{a}}\right)$ & $78\left(79^{a}\right)$ & 103 & 0.62 & 48 & 125 & 0.30 & 185 & 555 \\
\hline $\mathrm{Si}-34$ & 2074 & $135\left(137^{\mathrm{c}}\right)$ & $55\left(57^{c}\right)$ & $44\left(39^{c}\right)$ & 82 & 0.92 & 40 & 103 & 0.29 & 111 & 516 \\
\hline $\mathrm{Si}-46$ & 2098 & $140\left(135^{\mathrm{c}}\right)$ & $47\left(58^{c}\right)$ & $47\left(40^{c}\right)$ & 78 & 0.97 & 46 & 116 & 0.25 & 118 & 551 \\
\hline Ge-2 & 5566 & $134\left(130^{\mathrm{a}}\right)$ & $54\left(48^{a}\right)$ & $66\left(68^{a}\right)$ & 82 & 0.59 & 39 & 102 & 0.29 & 156 & 315 \\
\hline $\mathrm{Ge}-34$ & 4902 & 114 & 46 & 36 & 69 & 0.94 & 34 & 88 & 0.29 & 92 & 301 \\
\hline Ge-46 & 4953 & 115 & 37 & 40 & 63 & 0.96 & 39 & 97 & 0.24 & 101 & 320 \\
\hline
\end{tabular}

${ }^{a}$ Experiment, Ref. 31.

bTheory, Ref. 19.

'Theory, Ref. 33.

For diamond, simulations reproduce with accuracy experimental data (see Table IX). The differences for clathrates between previous calculations of elastic constants and ours simulations are small. The elastic constants of clathrates are always smaller (10-20\%) than diamond analogs.

From elastic constants, experimental sets of parameters such the bulk modulus $B_{o}$, the factor of anisotropy $C_{a}$, the shear modulus $G$, and the Young's modulus $Y$ along two main crystallographic directions ([100] and [111]) can be deduced according to the following relations (see Table IX):

$$
\begin{gathered}
B_{o}=\frac{C_{11}+2 C_{12}}{3}, \\
C_{a}=\frac{C_{11}-C_{12}}{2 C_{44}}, \\
G=\frac{C_{11}-C_{12}}{2}, \\
Y_{100}=\frac{\left(C_{11}+2 C_{12}\right)\left(C_{11}-C_{12}\right)}{C_{11}+C_{12}}, \\
\frac{1}{Y_{111}}=\frac{1}{Y_{100}}+\frac{C_{11}-C_{12}-2 C_{44}}{3 C_{44}\left(C_{11}-C_{12}\right)} .
\end{gathered}
$$

One can first note that $B_{o}$ calculated from a Murnaghan fit (see Table II) and from elastic constants (Table IX) are very close. The factor of anisotropy is close to 1 in clathrates, indicating that they can be viewed as strongly isotropic systems according to strains. The Poisson's ratio $\nu$, which quantifies the stability of a crystal against shear, are considerably smaller for carbon systems than for the other silicon/ germanium systems, which suggests that carbon clathrates should be relatively stable against shear. To conclude, one found that the Young's Modulus vary little in function to the crystallographic direction. These results could explain the good stability of clathrates under pressure.

\section{CONCLUSION}

We have reported first-principles study of free guest carbon clathrates properties C-34 and C-46, and compared them with silicon and germanium clathrates and diamond analogs. In spite of a similar $s p^{3}$ hybridization for the nine systems, we have shown that the lack of $p$ core states in carbon materials induces strong modification in vibrational properties comparatively to $\mathrm{Si}$ and $\mathrm{Ge}$. The pentagonal rings provide additional strong changes in electronic and vibrational properties.

The study of electronic states around the band gap shows a slight dependency on the element, which results in the same optical properties in silicon and carbon clathrates. We have shown that the vibrational properties of carbon phases differ from their $\mathrm{Si} / \mathrm{Ge}$ analog structures, whereas vibrational properties of $\mathrm{Si}$ and $\mathrm{Ge}$ systems can be relied through their atomic weight ratio. Raman and infrared spectra are displayed for an upcoming suitable experimental analysis. Thermodynamical properties $\left(S, F, C_{v}\right)$ are revealing to be less altered by crystallographic effect and that for a given element it is difficult to separate behaviors. It is only at high pressure that the evolution of lattice parameters of clathrates differs from diamond. The phase diagram calculated is found to be compatible with previous estimations. We conclude the paper with a presentation of elastic properties computed from acoustic slopes.

\section{ACKNOWLEDGMENTS}

Calculations have been performed at supercomputer facilities at Calmip CICT Toulouse (France). D.C. is indebted to P. Demont and D. Monceau for a critical reading of the manuscript. 
*damien.connetable@ensiacet.fr

${ }^{1}$ J. L. Cohn, G. S. Nolas, V. Fessatidis, T. H. Metcalf, and G. A. Slack, Phys. Rev. Lett. 82, 779 (1999).

${ }^{2}$ J. S. Tse, K. Uehara, R. Rousseau, A. Ker, C. I. Ratcliffe, M. A. White, and G. MacKay, Phys. Rev. Lett. 85, 114 (2000).

${ }^{3}$ L. Qiu, M. A. White, Z. Li, J. S. Tse, C. I. Ratcliffe, C. A. Tulk, J. Dong, and O. F. Sankey, Phys. Rev. B 64, 024303 (2001).

${ }^{4}$ G. S. Nolas, J.-M. Ward, J. Gryko, L. Qiu, and M. A. White, Phys. Rev. B 64, 153201 (2001).

${ }^{5}$ D. Connétable, V. Timoshevskii, E. Artacho, and X. Blase, Phys. Rev. Lett. 87, 206405 (2001).

${ }^{6}$ G. Benedek, M. Bernasconi, and A. Gambirasio, Phys. Status Solidi B 237, 296 (2003).

${ }^{7}$ M. Bernasconi, S. Gaito, and G. Benedek, Phys. Rev. B 61, 12689 (2000).

${ }^{8}$ D. Connétable, Phys. Rev. B 75, 125202 (2007).

${ }^{9}$ H. Kawaji, H. O. Horie, S. Yamanaka, and M. Ishikawa, Phys. Rev. Lett. 74, 1427 (1995).

${ }^{10}$ D. Connétable, V. Timoshevskii, B. Masenelli, J. Beille, J. Marcus, B. Barbara, A. M. Saitta, G.-M. Rignanese, P. Mélinon, S. Yamanaka, and X. Blase, Phys. Rev. Lett. 91, 247001 (2003).

${ }^{11}$ A. San-Miguel, P. Kéghélian, X. Blase, P. Mélinon, A. Perez, J. P. Itié, A. Polian, E. Reny, C. Cros, and M. Pouchard, Phys. Rev. Lett. 83, 5290 (1999).

${ }^{12}$ G. B. Adams, M. O'Keeffe, A. A. Demkov, O. F. Sankey, and Y.-M. Huang, Phys. Rev. B 49, 8048 (1994).

${ }^{13}$ C. A. Perottoni and J. A. H. da Jornada, J. Phys.: Condens. Matter 13, 5981 (2001).

${ }^{14}$ R. Nesper, K. Vogel, and P. E. Blöchl, Angew. Chem. 32, 701 (1993).

${ }^{15} \mathrm{G}$. Benedek and L. Colombo, Mater. Sci. Forum 232, 247 (1996); I. Spagnolatti, M. Bernasconi, and G. Benedek, Eur. Phys. J. B 34, 63 (2003).

${ }^{16}$ N. Rey, A. Munoz, P. Rodriguez-Hernández, and A. San-Miguel, J. Phys.: Condens. Matter 20, 215218 (2008).

${ }^{17}$ X. Blase, Phys. Rev. B 67, 035211 (2003).

${ }^{18}$ D. Connétable and X. Blase, Appl. Surf. Sci. 226, 289 (2004).

${ }^{19}$ X. Blase, P. Gillet, A. San Miguel, and P. Melinon, Phys. Rev. Lett. 92, 215505 (2004).

${ }^{20}$ F. Zipoli, M. Bernasconi, and G. Benedek, Phys. Rev. B 74, 205408 (2006).

${ }^{21}$ A. San-Miguel and P. Toulemonde, High Press. Res. 25, 159 (2005).

${ }^{22}$ P. Mélinon, P. Kéghélian, X. Blase, J. Le Brusc, A. Perez, E. Reny, C. Cros, and M. Pouchard, Phys. Rev. B 58, 12590 (1998).

${ }^{23}$ P. Giannozzi, S. Baroni, N. Bonini, M. Calandra, R. Car, C. Cavazzoni, D. Ceresoli, G. L. Chiarotti, M. Cococcioni, I. Dabo, A. Dal Corso, S. Fabris, G. Fratesi, S. de Gironcoli, R. Gebauer, U. Gerstmann, C. Gougoussis, A. Kokalj, M. Lazzeri, L. MartinSamos, N. Marzari, F. Mauri, R. Mazzarello, S. Paolini, A. Pasquarello, L. Paulatto, C. Sbraccia, S. Scandolo, G. Sclauzero, A. P. Seitsonen, A. Smogunov, P. Umari, and R. M. Wentzcovitch, J. Phys.: Condens. Matter 21, 395502 (2009).

${ }^{24}$ J. P. Perdew and A. Zunger, Phys. Rev. B 23, 5048 (1981).

${ }^{25}$ D. M. Ceperley and B. J. Alder, Phys. Rev. Lett. 45, 566 (1980).

${ }^{26}$ N. Troullier and J. L. Martins, Phys. Rev. B 43, 1993 (1991); Solid State Commun. 74, 613 (1990).

${ }^{27}$ L. Kleinman and D. M. Bylander, Phys. Rev. Lett. 48, 1425
(1982).

${ }^{28}$ H. J. Monkhorst and J. D. Pack, Phys. Rev. B 13, 5188 (1976).

${ }^{29}$ S. Baroni and R. Resta, Phys. Rev. B 33, 7017 (1986).

${ }^{30}$ S. Baroni, P. Giannozzi, and A. Testa, Phys. Rev. Lett. 58, 1861 (1987).

${ }^{31}$ C. Kittel, Introduction to Solid State Physics (Wiley, New York, 1996).

${ }^{32}$ G. S. Nolas, M. Beekman, J. Gryko, G. A. Lamberton, T. M. Tritt, and P. F. McMillan, Appl. Phys. Lett. 82, 910 (2003).

${ }^{33}$ K. Moriguchi, S. Munetoh, A. Shintani, and T. Motooka, Phys. Rev. B 64, 195409 (2001).

${ }^{34}$ J. Dong and O. F. Sankey, J. Phys.: Condens. Matter 11, 6129 (1999).

${ }^{35}$ A. M. Guloy, R. Ramlau, Z. Tang, W. Schnelle, M. Baitinger, and Y. Grin, Nature (London) 443, 320 (2006).

${ }^{36}$ K. Moriguchi, S. Munetoh, and A. Shintani, Phys. Rev. B 62, 7138 (2000).

${ }^{37}$ F. D. Murnaghan, Proc. Natl. Acad. Sci. U.S.A. 30, 244 (1944).

${ }^{38}$ J. Gryko, P. F. McMillan, R. F. Marzke, G. K. Ramachandran, D. Patton, S. K. Deb, and O. F. Sankey, Phys. Rev. B 62, R7707 (2000).

${ }^{39}$ J. Zhao, A. Buldum, J. P. Lu, and C. Y. Fong, Phys. Rev. B 60, 14177 (1999).

${ }^{40}$ G. S. Nolas, C. A. Kendziora, J. Gryko, J. Dong, C. W. Myles, A. Poddar, and O. F. Sankey, J. Appl. Phys. 92, 7225 (2002).

${ }^{41}$ X. Tang, J. Dong, P. Hutchins, O. Shebanova, J. Gryko, P. Barnes, J. K. Cockroft, M. Vickers, and P. F. McMillan, Phys. Rev. B 74, 014109 (2006).

${ }^{42}$ M. Menon, E. Richter, and K. R. Subbaswamy, Phys. Rev. B 56, 12290 (1997).

${ }^{43}$ D. L. Rousseau, R. P. Bauman, and S. P. S. Porto, J. Raman Spectrosc. 10, 253 (1981).

${ }^{44}$ Y. Guyot, B. Champagnon, E. Reny, C. Cros, M. Pouchard, P. Melinon, A. Perez, and I. Gregora, Phys. Rev. B 57, R9475 (1998).

${ }^{45}$ It is interesting to note that vibrational properties of tin clathrates can too be deduced from germanium thanks a atomic weight ratio with a good accuracy.

${ }^{46}$ P. Giannozzi, S. de Gironcoli, P. Pavone, and S. Baroni, Phys. Rev. B 43, 7231 (1991).

${ }^{47}$ P. Pavone, K. Karch, O. Schütt, W. Windl, D. Strauch, P. Giannozzi, and S. Baroni, Phys. Rev. B 48, 3156 (1993).

${ }^{48}$ M. Lazzeri and F. Mauri, Phys. Rev. Lett. 90, 036401 (2003).

${ }^{49}$ D. Porezag and M. R. Pederson, Phys. Rev. B 54, 7830 (1996).

${ }^{50}$ J. Dong, O. F. Sankey, and G. Kern, Phys. Rev. B 60, 950 (1999).

${ }^{51}$ J. C. Li, C. L. Wang, W. X. Wang, H. Peng, R. Z. Zhang, M. L. Zhao, J. Liu, J. L. Zhang, and L. M. Mei, J. Appl. Phys. 105, 043503 (2009).

${ }^{52}$ G. A. Slack and S. F. Bartram, J. Appl. Phys. 46, 89 (1975).

${ }^{53}$ K. Biswas, C. W. Myles, M. Sanati, and G. S. Nolas, J. Appl. Phys. 104, 033535 (2008).

${ }^{54}$ J. Xie, S. P. Chen, J. S. Tse, S. de Gironcoli, and S. Baroni, Phys. Rev. B 60, 9444 (1999).

${ }^{55}$ B. B. Pate, I. Lindau, and W. E. Spicer, in Proceedings of the 17th International Conference on the Physics of Semiconductors, San Francisco, 1984, edited by J. D. Chadi and W. A. Harrison (Springer-Verlag, New York, 1985), p. 1181. 